\title{
Simple modules and their essential extensions for skew polynomial rings
}

\author{
Ken Brown ${ }^{1}$. Paula A. A. B. Carvalho ${ }^{2}$. Jerzy Matczuk ${ }^{3}$
}

Received: 10 June 2017 / Accepted: 9 July 2018 / Published online: 21 August 2018

(c) The Author(s) 2018

\begin{abstract}
Let $R$ be a commutative Noetherian ring and $\alpha$ an automorphism of $R$. This paper addresses the question: when does the skew polynomial ring $S=R[\theta ; \alpha]$ satisfy the property $(\diamond)$, that for every simple $S$-module $V$ the injective hull $E_{S}(V)$ of $V$ has all its finitely generated submodules Artinian. The question is largely reduced to the special case where $S$ is primitive, for which necessary and sufficient conditions are found, which however do not between them cover all possibilities. Nevertheless a complete characterisation is found when $R$ is an affine algebra over a field $k$ and $\alpha$ is a $k$-algebra automorphism-in this case $(\diamond)$ holds if and only if all simple $S$-modules are finite dimensional over $k$. This leads to a discussion, involving close study of some families of examples, of when this latter condition holds for affine $k$-algebras $S=R[\theta ; \alpha]$. The paper ends with a number of open questions.
\end{abstract}

Keywords Injective module $\cdot$ Noetherian ring $\cdot$ Simple module $\cdot$ Skew polynomial ring

Mathematics Subject Classification $16 \mathrm{D} 50 \cdot 16 \mathrm{P} 40 \cdot 16 \mathrm{~S} 35$

\section{Introduction}

A Noetherian ring $S$ whose simple modules have the property that their finitely generated essential extensions are Artinian is said to satisfy property $(\diamond)$. For commutative rings $S$ the validity of $(\diamond)$ is due to Matlis, proved in his famous 1958 paper [30]; a brief survey of work on this topic in the years since then is given below, in Sect. 1.3. This paper focusses on $(\diamond)$

\footnotetext{
$凶 \quad$ Ken Brown

Ken.Brown@glasgow.ac.uk

Paula A. A. B. Carvalho

pbcarval@fc.up.pt

Jerzy Matczuk

jmatczuk@mimuw.edu.pl

1 School of Mathematics and Statistics, University of Glasgow, Glasgow G12 8SQ, Scotland

2 Departamento de Matemática, Faculdade de Ciências, Universidade do Porto, Rua Campo Alegre 687, 4169-007 Porto, Portugal

3 Institute of Mathematics, Warsaw University, Banacha 2, 02-097 Warsaw, Poland
} 
for the skew polynomial rings $S=R[\theta ; \alpha]$, where $R$ is a commutative Noetherian ring and $\alpha$ is an automorphism of $R$, with the indeterminate $\theta$ satisfying the relations $\theta r=\alpha(r) \theta$ for all $r \in R$. When such a skew polynomial ring $S$ satisfies $(\diamond)$ turns out to be a surprisingly subtle question, which we do not completely settle here, and which leads naturally to other fundamental representation-theoretic questions concerning these rings.

We are led to pay particular attention to two separate but overlapping cases-first, when $S$ is a primitive ring; and second, when $R$ is an affine algebra over a field. We outline our main results for these two settings in Sects. 1.1 and 1.2 respectively. Here and throughout, given a ring $R$ and an $R$-module $V, E_{R}(V)$ will denote the $R$-injective hull of $V$.

\subsection{When $S$ is primitive}

Using relatively standard methods involving the second layer condition we show that, for every commutative Noetherian ring $R, S=R[\theta ; \alpha]$ satisfies $(\diamond)$ if and only if $E_{S}(V)$ is locally Artinian for every simple $S$-module $V$ whose annihilator $Q$ is induced from $R$. Here, "induced from $R$ " means that $Q=(Q \cap R) S$. This reduction is achieved in Corollary 3.5, thereby focussing attention on the case where $S=R[\theta ; \alpha]$ is primitive, since $S /(Q \cap R) S \cong$ $\bar{R}[\theta ; \bar{\alpha}]$, where $\bar{R}=R / Q \cap R$ and $\bar{\alpha}$ denotes the automorphism of $\bar{R}$ induced by $\alpha$.

Making heavy use of the characterisation of primitive skew polynomial rings [29], we prove:

Theorem 1.1 Let $R$ be a commutative Noetherian ring, let $\alpha$ be an automorphism of $R$, and let $S=R[\theta ; \alpha]$. Suppose that $S$ is primitive.

(a) If $R$ has Krull dimension 0 then $S$ satisfies $(\diamond)$.

(b) Suppose that $R$ contains an uncountable field. Suppose also that either $R$ has Krull dimension at least 2 , or $\operatorname{Spec}(R)$ is uncountable. Then $S$ does not satisfy $(\diamond)$.

The above is Theorem 5.4; its proof occupies Sects. 4, 5. Clearly, the above necessary and sufficient conditions don't exhaust all possibilities, and we leave the closure of the gap between (a) and (b) as one of a number of open questions raised by the paper.

\subsection{When $R$ is affine}

Theorem 1.1 is however sufficient to settle the case where $R$ is a finitely generated algebra over an uncountable field $k$, with $\alpha$ a $k$-algebra automorphism:

Theorem 1.2 Let $k$ be an uncountable field and $R$ an affine $k$-algebra, and let $\alpha$ be a $k$-algebra automorphism of $R$. Let $S=R[\theta ; \alpha]$. Then the following are equivalent:

(a) S satisfies $(\diamond)$;

(b) all simple $S$-modules are finite dimensional $k$-vector spaces.

This is Theorem 6.1. The direction $(b) \Rightarrow(a)$ follows from known considerations based on the second layer condition, and doesn't require the cardinality hypothesis on $k$. In fact, following very welcome input from Jason Bell [2] and the application of deep model-theoretic work [20], Theorem 6.1 can be extended to countable fields, at least when $k$ has characteristic 0-see Remark(ii) following Theorem 6.1.

Theorem 1.2 begs the obvious question: for which commutative affine $k$-algebras $R$ and $k$-algebra automorphisms $\alpha$ are all the simple $S$-modules finite-dimensional? This question 
seems to be hard, but we can at least answer it in the most obvious special case, namely when $R$ is a polynomial algebra and $\alpha$ is a linear automorphism:

Theorem 1.3 Let $k$ be a field, $t$ a positive integer, $V$ a vector space over $k$ with basis $\left\{x_{1}, \ldots, x_{t}\right\}$ and $\alpha \in \mathrm{GL}(t, k)$ an automorphism of $V$. Let $R=k\left[x_{1}, \ldots, x_{t}\right]$, so that $\alpha$ induces a $k$-algebra automorphism of $R$, also denoted by $\alpha$. Then $S:=R[\theta ; \alpha]$ satisfies $(\diamond)$ if and only if all simple $S$-modules are finite dimensional if and only if $|\alpha|<\infty$.

Beyond this positive result, we demonstrate the complexity of the above question by means of detailed analysis of some families of examples in Sect. 7. These include an example, Example 7.4 , originally studied by Jordan [25], giving an affine domain in characteristic 0 where all the simple $S$-modules have finite dimension, but $\alpha$ has infinite order. Using deep results on group algebras, we also provide many similar examples in positive characteristic (Examples 7.3). Most notably, we undertake a detailed study of the algebras $S_{\mathbb{C}, t, \alpha}=\mathbb{C}\left[x_{1}, \ldots, x_{t}\right][\theta ; \alpha]$ when $t=1$ or 2 and $\alpha$ is an arbitrary $\mathbb{C}$-algebra automorphism. But even for $t=2$ our analysis is incomplete, and leads to delicate issues having connections to dynamical systems and to algebraic geometry.

\subsection{Historical background}

For commutative Noetherian rings $(\diamond)$ is an immediate consequence of the Artin-Rees property, formally recorded as part of Matlis's seminal 1958 paper [30] on injective modules over such rings. In 1959 Philip Hall proved $(\diamond)$ for group rings $R G$ of finitely generated nilpotent groups $G$, provided $R$ is either $\mathbb{Z}$ or a locally finite field. In 1974 this result was extended, independently by Jategaonkar [23] and Roseblade [39], to polycyclic-by-finite groups $G$, building on earlier celebrated work of Roseblade [38] on the finite dimensionality of the simple $R G$-modules for these group rings. Hall and Roseblade were motivated by applications to the structure of finitely generated soluble groups.

Motivated by applications to Jacobson's conjecture, Jategaonkar proved in 1974 [22] that $(\diamond)$ is satisfied by fully bounded Noetherian rings, thus incorporating Noetherian rings satisfying a polynomial identity (PI) and so generalising the commutative case. Musson [32] gave the first examples of Noetherian rings for which $(\diamond)$ fails, by showing that the group algebra $k G$ of a polycyclic-by-finite group $G$ over a field $k$ which is not locally finite satisfies $(\diamond)$ only if $G$ is abelian-by-finite, that is only if $k G$ satisfies a PI. In so doing he thus delineated the limits of the earlier results of Hall and Roseblade.

More recent work has discussed $(\diamond)$ for differential operator rings [7], [40], down-up algebras [6] and quantised Weyl algebras [8]. Musson [34] gives a brief survey of results on property $(\diamond)$ up to 2010 .

\subsection{Layout}

Preliminary observations and notation regarding property $(\diamond)$ and skew polynomial rings are in Sect. 2. Section 3 contains a summary of the necessary background on the second layer condition, leading up to Corollary 3.5, which essentially allows us to focus on the case where $S=R[\theta ; \alpha]$ is a primitive ring. The analysis of primitive skew polynomial rings is contained in Sects. 4 and 5: the key result of Sect. 4 is the construction of a faithful simple $S$-module whose injective hull is not locally Artinian when $R$ is an $\alpha$-simple domain which is not a field. This is Proposition 4.10, which then allows us to deduce Theorem 1.1 (= Theorem 5.4). In Sect. 6 Theorem 1.1 is applied in the setting where $R$ is an affine algebra over the field 
$k$ and $\alpha$ is an algebra automorphism, to deduce Theorems 1.2 and 1.3 (= Theorems 6.1 and 6.2). Section 7 is devoted to a careful analysis of the simple modules and the prime spectra of a number of examples, and families of examples, of skew polynomial algebras over a commutative affine Noetherian domain $R$. These examples may well have interest beyond the immediate question at hand, namely the validity of $(\diamond)$. Finally, in Sect. 8 we gather together and briefly discuss the open questions which have arisen in the course of this work.

All rings considered are associative with identity and all modules are unitary and are right modules unless stated otherwise.

\section{Preliminaries}

\subsection{Formulation and preservation of $(\diamond)$}

Recall that a module $M$ is a subdirect product of a family of modules $\left\{F_{\lambda}\right\}$ if there exists an embedding $\iota: M \rightarrow \prod_{\lambda \in \Lambda} F_{\lambda}$ into the product of the modules $F_{\lambda}$ such that, for each projection $\pi_{\mu}: \prod_{\lambda \in \Lambda} F_{\lambda} \rightarrow F_{\mu}$, the composition $\pi_{\mu} \iota$ is surjective. The following lemma is due to Hatipoğlu and Lomp, [17].

Lemma 2.1 [17, Lemma 2.1] Given a ring $R$, the following conditions are equivalent:

(a) $R$ satisfies $(\diamond)$;

(b) every right $R$-module is a subdirect product of locally Artinian modules;

(c) every finitely generated right $R$-module is a subdirect product of Artinian modules.

Recall that a ring extension $T \subseteq S$ is called a finite normalizing extension if there exists a finite set $\left\{s_{1}, \ldots, s_{n}\right\}$ of elements of $S$ such that $S=\sum_{i=0}^{n} s_{i} T$, with $s_{i} T=T s_{i}$, for $i=1, \ldots, n$. Part (a) of the next result is due to Hatipoğlu and Lomp, [17, Proposition 2.2], while (b) is adapted from the work of Hirano [19, Theorems 1.8, 1.11].

Proposition 2.2 Let $S$ be a finite normalizing extension of a ring $T$.

(a) If $T$ satisfies $(\diamond)$, then so does $S$.

(b) Assume also that $T$ is Noetherian and a direct summand of $S$ as a left $T$-module. If $S$ satisfies $(\diamond)$, then so does $T$.

(c) If $I$ is an ideal of $S$ and $S$ satisfies $(\diamond)$, then so does $S / I$.

Proof (a) See [17, Proposition 2.2].

(b) Let $M$ be a finitely generated right $T$-module, so $M \otimes_{T} S$ is a finitely generated right $S$-module. By hypothesis and Lemma 2.1 there exists a family $\left\{M_{\lambda}\right\}$ of $S$-submodules of $M \otimes_{T} S$ such that each $\left(M \otimes_{T} S\right) / M_{\lambda}$ is Artinian and $\bigcap_{\lambda} M_{\lambda}=0$. Since $T$ is a direct summand of $S$ as a left $T$-module, $M$ can be identified with a right $T$-submodule of $M \otimes_{T} S$. Fix $\lambda$. Then $M /\left(M \cap M_{\lambda}\right)$ is isomorphic to a $T$-submodule of $\left(M \otimes_{T} S\right) / M_{\lambda}$. By [12, Theorem 4], $\left(M \otimes_{T} S\right) / M_{\lambda}$ is Artinian as an $S$-module if and only if it is Artinian as a $T$-module. Now, note that $\bigcap_{\lambda}\left(M \cap M_{\lambda}\right)=0$, so the result follows from Lemma 2.1(c).

(c) This is trivial, since $E_{S / I}(V) \subseteq E_{S}(V)$ for any $S / I$-module $V$. 


\subsection{Skew polynomial algebras of automorphism type}

Let $R$ be a ring and let $\alpha$ be an automorphism of $R$. The skew polynomial ring of automorphism type, $S:=R[\theta ; \alpha]$, is the ring of polynomials in $\theta$ with coefficients in $R$ subject to the relation $\theta r=\alpha(r) \theta$ for all $r \in R$.

We shall maintain the notation $S, R, \theta, \alpha$ throughout the paper.

Often, $R$ will in addition be commutative or Noetherian, but we will nevertheless state these hypotheses as required in our results, for emphasis. A right ideal $I$ of $R$ is said to be $\alpha$-stable if $\alpha(I)=I$. We say that $R$ is $\alpha$-simple if (0) and $R$ are the only $\alpha$-stable ideals of $R$. An $\alpha$-stable ideal $P$ of $R$ is $\alpha$-prime if, for all $\alpha$-stable ideals $I$ and $J$ of $R, I J \subseteq P$ implies that $I \subseteq P$ or $J \subseteq P$. The ring $R$ is said to be $\alpha$-prime if its ideal (0) is $\alpha$-prime, and is called $\alpha$-simple if its only $\alpha$-stable ideals are (0) and $R$.

The following well known facts can be found in [31, §10.6].

Lemma 2.3 Let $R$ be a right Noetherian ring, $\alpha$ an automorphism of $R$ and $S=R[\theta ; \alpha]$.

(a) A right ideal I of $R$ is $\alpha$-stable if and only if $\alpha(I) \subseteq I$.

(b) If I is an $\alpha$-stable ideal of $R$, then IS is an ideal of $S$ and the ring $S / I S$ is isomorphic to $(R / I)[\theta ; \bar{\alpha}]$, where $\bar{\alpha}$ is the automorphism of $R / I$ induced by $\alpha$. The ideal IS is prime if and only if I is $\alpha$-prime.

(c) If $P$ is a prime ideal of $S$ such that $\theta \notin P$ then $P \cap R$ is an $\alpha$-prime ideal of $R$.

(d) If $P$ is an $\alpha$-prime ideal of $R$, then there exists $n \in \mathbb{N}$ and a minimal prime $Q$ over $P$ such that $P=\bigcap_{i=0}^{n} \alpha^{i}(Q)$.

A convenient mechanism to construct interesting simple $S$-modules is as follows.

Lemma 2.4 Suppose that $u$ is a central unit of a right Noetherian ring $R$ and $S=R[\theta ; \alpha]$.

(a) The lattice of right $\alpha$-ideals of $R$ is isomorphic to the lattice of $S$-submodules of $S /(u-\theta) S$.

Suppose additionally in (b) and (c) that $R$ is commutative.

(b) The right $S$-module $S /(u-\theta) S$ is simple if and only if $R$ is $\alpha$-simple.

(c) Suppose that $R$ is also a domain with no proper idempotent ideals; for example, $R$ could be a Noetherian domain. Then $S /(u-\theta) S$ is an Artinian right $S$-module if and only if $R$ is $\alpha$-simple.

Proof (a) Notice that, for any element $r \in R$,

$$
r \theta=\theta \alpha^{-1}(r)=-(u-\theta) \alpha^{-1}(r)+u \alpha^{-1}(r) .
$$

Let $N$ be an $\alpha$-stable right ideal of $R$. Then (1) and the centrality of $u$ in $R$ yield that $J:=(u-\theta) S+N$ is a right ideal of $S$.

Let $J$ be a right ideal of $S$ with $(u-\theta) S \subseteq J$. Let $w \in J$. Using division by the monic polynomial $u-\theta$ we can find $h \in S$ and $r \in R$ such that $w=(u-\theta) h+r$ and $J=(u-\theta) S+N$ follows for $N:=J \cap R$. Moreover, applying (1) with $r \in N$ and using our assumptions on $u$, it follows that $\alpha^{-1}(N) \subseteq N$. By Lemma 2.3(a), $N$ is an $\alpha$-stable right ideal of $R$. This completes the proof of (a).

(b) This is a direct consequence of (a). 
(c) Suppose that $S /(u-\theta) S$ is Artinian as a right $S$-module. Let $I$ be a proper $\alpha$-stable ideal of $R$. It follows from (a) that there exists $n \in \mathbb{N}$ such that $I^{n}=I^{2 n}$. The stated hypotheses force $I$ to be (0), so $R$ is $\alpha$-simple. The converse is given by (b). The Krull Intersection Theorem [10, Corollary 5.4] ensures that Noetherian domains satisfy the stated hypothesis.

\section{Reduction to the primitive case}

\subsection{The second layer condition}

Given a non-zero module $M$ over a right Noetherian ring $T$, an affiliated submodule of $M$ is a submodule of the form $\operatorname{Ann}_{M}(P)=\{m \in M: m P=0\}$, where $P$ is an ideal of $T$ which is maximal amongst the annihilators of non-zero submodules of $M$. It is easy to see that such an ideal $P$ is a prime ideal of $T$, [16, Proposition 3.12]. An affiliated series for $M$ is a series $0=M_{0} \subset M_{1} \subset \cdots \subset M_{n}=M$ of submodules of $M$, such that for each $i \in\{1, \ldots, n\}$, $M_{i} / M_{i-1}$ is an affiliated submodule of $M / M_{i-1}$. The ideals $P_{i}:=\operatorname{Ann}_{T}\left(M_{i} / M_{i-1}\right)$ are called the affiliated primes of $M$ with respect to the given series. Full details are in [16, Chapter 8], for example.

We briefly recall here the key ideas we need from the theory of prime links for Noetherian rings. For more details, see for example [16, Chapter 12], [24]. Let $T$ be a Noetherian ring and $M$ a finitely generated right $T$-module. Suppose that

$$
0 \subset U \subset M
$$

is an affiliated series of $M$, and suppose that $U$ is an essential submodule of $M$, with corresponding affiliated prime ideals $Q$ and $P$, so that $U Q=0=(M / U) P$. To understand the possible relations between the modules $U$ and $M / U$, normalise (2) by replacing $M$ if necessary with a submodule $M^{\prime}$ of $M$ properly containing $U$ such that $I:=\operatorname{Ann}_{T}\left(M^{\prime}\right)$ is maximal amongst the annihilators of those submodules of $M$ which properly contain $U$. Notice that $P Q \subseteq I$. With this normalisation, we continue to write $M$ for the replacement module.

Then, by Jategaonkar's so-called Main Lemma, [16, Theorem 12.1], [24], there are two possibilities: either

(a) $P Q \subseteq I \subset P \cap Q$, and $(P \cap Q) / I$ is torsion-free as a left $T / P$-module and as a right $T / Q$-module; or

(b) $P \subset Q$ and $M P=0$.

In partial converse, if (a) holds for primes $P$ and $Q$ of $T$, then a right $T$-module $M$ exists as above, with $U$ [resp. $M / U$ ] being $T / Q$ [resp. $T / P$ ]-torsion-free, [16, Theorem 12.2]. If case (a) holds, we say that there is a link from $P$ to $Q$, and we write $P \rightsquigarrow Q$. If case (a) holds and $U$ is $T / Q$-torsion-free, then $M / U$ is $T / P$-torsion-free. On the other hand, if (b) holds, then both $M$ and $M / U$ are $T / P$-torsion.

A Noetherian ring $T$ is said to satisfy the (right) strong second layer condition (s.s.l.c.) if, for every prime ideal $Q$ of $T$, only case (a) can occur in the setting of (2). The formally weaker (right) second layer condition (s.l.c.) holds for $T$ if, for all primes $Q$, only case (a) occurs when $U$ is in addition required to be $T / Q$-torsion-free.

Those Noetherian rings satisfying the s.s.l.c. form an important and large subclass. For our purposes, the key result in this direction is the following proposition. Recall (for example, from [16, page 224]) that a Noetherian ring $T$ is $A R$-separated if, for every prime ideal $Q$ of 
$T$ and ideal $I$ with $Q \subset I \subset T$, there is an ideal $J$ of $T$ with $Q \subset J \subseteq I$ such that $J / Q$ has the Artin-Rees property in $T / Q$.

Proposition 3.1 [16, Theorem 13.4] If the Noetherian ring $T$ is AR-separated, then it satisfies the s.s.l.c.

For example, Noetherian rings satisfying a polynomial identity and enveloping algebras of finite dimensional solvable Lie algebras are AR-separated, [24]. Of more relevance for us, however, is:

Proposition 3.2 [36] Let $R$ be a commutative Noetherian ring, $\alpha \in \operatorname{Aut}(R)$ and $S=R[\theta ; \alpha]$.

(a) Then $S$ is AR-separated, and hence satisfies s.s.l.c.

(b) Let $P$ be a prime ideal of $S$ such that $(P \cap R) S=P$. Then $P$ has the Artin-Rees property. In particular, if $P \rightsquigarrow Q$ or $Q \rightsquigarrow P$, then $Q=P$.

\subsection{The second layer condition and $(\diamond)$}

Lenagan's Lemma, [16, Theorem 7.11], guarantees that if $T$ is a Noetherian ring and $P$ and $Q$ are prime ideals of $T$ with $P \rightsquigarrow Q$, then $T / P$ is Artinian if and only if $T / Q$ is Artinian. From this and Jategaonkar's Main Lemma the following well-known consequence follows easily:

Proposition 3.3 Let $T$ be a Noetherian ring satisfying the s.l.c. and let $V$ be a simple right $T$-module with $Q:=\operatorname{Ann}_{T}(V)$. Suppose that $T / Q$ is Artinian. Then every finitely generated essential extension of $V$ is Artinian.

We are now in a position to deal with property $(\diamond)$ for many simple modules over skew polynomial rings.

Theorem 3.4 Let $R$ be a commutative Noetherian ring, $\alpha \in A u t(R)$ and $S=R[\theta ; \alpha]$. Let $V$ be a simple right $S$-module and let $Q=a_{n} n_{S}(V)$. If $(Q \cap R) S \subset Q$, then $S / Q$ is Artinian and (hence) every finitely generated essential extension of $V$ is Artinian.

Proof Suppose first that $\theta \in Q$. Then $Q / \theta S$ is a primitive ideal of the commutative ring $S / \theta S \simeq R$, so $S / Q$ is a field. Therefore the desired property of $V$ follows from Propositions 3.2(a) and 3.3.

Suppose now that $\theta \notin Q$ and $(Q \cap R) S \subset Q$. Then $Q \cap R$ is $\alpha$-prime by Lemma 2.3(c). By [21, Theorem 4.3] the order of the automorphism of $R /(Q \cap R)$ induced by $\alpha$ is finite. Hence, by [9, Corollary 10], $S /(Q \cap R) S \simeq(R /(Q \cap R))\left[\theta ;\left.\alpha\right|_{R /(Q \cap R)}\right]$ is a ring satisfying a polynomial identity. By Kaplansky's Theorem [4, Theorem I.13.3] applied to the primitive ideal $Q /(Q \cap R) S$ of $S /(Q \cap R) S$, we deduce once again that $S / Q$ is Artinian. Thus, again, $E_{S}(V)$ is locally Artinian by Propositions 3.2(a) and 3.3.

The following corollary of Theorem 3.4 in large part reduces the analysis of $(\diamond)$, for skew polynomial rings $S$ of automorphism type, to the case where $S$ is a primitive ring. Recall that a Noetherian ring $T$ is polynormal if for every pair of distinct ideals $I$ and $J$ of $T$ with $I \subset J$, there is an element $a \in J \backslash I$ such that $a S+I=S a+I$.

Corollary 3.5 Let $R$ be a commutative Noetherian ring, $\alpha \in \operatorname{Aut}(R)$ and $S=R[\theta ; \alpha]$. Consider the following statements: 
(a) $S$ satisfies $(\diamond)$;

(b) $E_{S}(V)$ is locally Artinian for every simple right $S$-module $V$ whose annihilator $Q$ satisfies $Q=(Q \cap R) S$;

(c) every primitive factor of $S$ of the form $S /(P \cap R) S$ satisfies $(\diamond)$.

Then $(a)$ and $(b)$ are equivalent and imply $(c)$. If additionally either $S$ is polynormal or every primitive ideal $P$ of $S$ of the form $P=(P \cap R) S$ is generated by a normal element, then all the above statements are equivalent.

Proof $(\mathrm{a}) \Leftrightarrow(\mathrm{b})$ is a consequence of Theorem 3.4. That $(\mathrm{a}) \Rightarrow(\mathrm{c})$ follows from Proposition 2.2(c).

If $S$ satisfies one of the additional hypotheses then (c) $\Rightarrow$ (a) follows from Theorem 3.4 combined with [24, Theorem 9.3.4]; see also [5, Lemmas 6.1 and 6.3].

\section{The primitive case}

\subsection{Primitive skew polynomial rings}

We begin by recalling the results of [29], where Leroy and Matczuk presented necessary and sufficient conditions for the primitivity of $S=R[\theta ; \alpha]$; see also [25].

Definition 4.1 Given a ring $T$ and $\alpha \in \operatorname{Aut}(T), T$ is $\alpha$-special if there is an element $a$ of $T$ such that the following conditions are satisfied.

(a) For all $n \geq 1, N_{n}^{\alpha}(a):=a \alpha(a) \ldots \alpha^{n-1}(a) \neq 0$.

(b) For every non-zero $\alpha$-stable ideal $I$ of $T$, there exists $n \geq 1$ such that $N_{n}^{\alpha}(a) \in I$.

When this occurs, the element $a$ is called an $\alpha$-special element.

From the definition it follows easily that an $\alpha$-special ring is $\alpha$-prime. Clearly, an $\alpha$-simple ring is $\alpha$-special, with 1 as $\alpha$-special element in this case. However, there are $\alpha$-special rings which are not $\alpha$-simple. Consider, for example, the ring $R$ of formal power series $k[[X]]$, where $k$ is a field containing an element $q$ which is not a root of unity. Let $\alpha$ be the $k$-algebra automorphism defined by setting $\alpha(X):=q X$. Then $X$ is an $\alpha$-special element of $R$, but $\langle X\rangle$ is a proper $\alpha$-ideal of $R$.

Here is the characterisation of primitivity:

Theorem 4.2 [29, Theorem 3.10] Let $R$ be a commutative Noetherian ring and let $\alpha \in$ $\operatorname{Aut}(R)$. Then $S=R[\theta ; \alpha]$ is primitive if and only if $R$ is $\alpha$-special and $\alpha$ has infinite order.

In the proof of Theorem 4.2, given a Noetherian PI $\operatorname{ring} R$ and an automorphism $\alpha$ of $R$ with $\alpha$-special element $a$, the authors build a simple faithful module over $S=R[\theta ; \alpha]$ of the form $S / M$, where $M$ is a maximal right ideal of $S$ containing $(1-a \theta) S$. Following similar ideas, we show in the proposition below that, when $R$ is commutative, $(1-a \theta) S$ is actually a maximal right ideal; this will be important for us in the sequel. Note also that the proposition provides a proof that the conditions on $\alpha$ in Theorem 4.2 are sufficient for primitivity of $S$ when $R$ is commutative.

Proposition 4.3 Let $R$ be a commutative Noetherian ring and let $\alpha \in A u t(R)$ be such that $R$ is $\alpha$-special with a an $\alpha$-special element of $R$. Let $S=R[\theta ; \alpha]$. Then $(1-a \theta) S$ is a maximal right ideal of $S$. If in addition $\alpha$ has infinite order, then $V:=S /(1-a \theta) S$ is a faithful right $S$-module. 


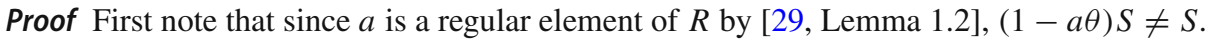
Let $J$ be a right ideal of $S$ such that $M:=(1-a \theta) S \subset J$. We claim that

$$
J \cap R \neq(0) .
$$

Let $n \geq k$ and let

$$
f=a_{n} \theta^{n}+\cdots+a_{k} \theta^{k} \in J \backslash(1-a \theta) S,
$$

with $f$ of shortest possible length $\ell(f):=n-k$. Suppose that $\ell(f)>0$. Then, since $1 \equiv a \theta(\bmod M)$,

$$
a_{k} \theta^{k} \equiv a \theta a_{k} \theta^{k} \equiv a \alpha\left(a_{k}\right) \theta^{k+1}(\bmod M) .
$$

Thus, $f \equiv g(\bmod M)$, where

$$
g:=a_{n} \theta^{n}+\cdots+\left(a_{k+1}+a \alpha\left(a_{k}\right)\right) \theta^{k+1},
$$

so that $\ell(g)<\ell(f)$. This contradicts our choice of $f$, and so $\ell(f)=0$. That is, $0 \neq a_{n} \theta^{n} \in$ $J$. But now $J$ also contains $\alpha^{-1}\left(a_{n}\right) \theta^{n-1}$, because

$$
a_{n} \theta^{n} \alpha^{-n}(a)=a a_{n} \theta^{n}=a \theta \alpha^{-1}\left(a_{n}\right) \theta^{n-1} \equiv \alpha^{-1}\left(a_{n}\right) \theta^{n-1}(\bmod M) .
$$

This implies that $\alpha^{-n}\left(a_{n}\right) \in J$, so (3) holds.

Next, observe that $J \cap R$ is an $\alpha$-stable ideal of $R$, since, if $r \in J \cap R$, then

$$
\alpha^{-1}(r) \equiv a \theta \alpha^{-1}(r)=r \theta \alpha^{-1}(a)(\bmod M),
$$

so that $\alpha^{-1}(r) \in J$. Therefore, as $a$ is an $\alpha$-special element of $R$ and $J \cap R$ is non-zero and $\alpha$ stable, there exists $k \in \mathbb{N}$ such that $(a \theta)^{k} \in J$. But then $(a \theta)^{k-1}=(1-a \theta)(a \theta)^{k-1}+(a \theta)^{k}$ and so $(a \theta)^{k-1} \in J$. It follows that $1 \in J$ and $(1-a \theta) S$ is maximal, as required.

To prove the last statement, assume that $\alpha$ is of infinite order. Let $P=\operatorname{Ann}_{S}(V)$. Then $P \subseteq(1-a \theta) S$, so $P \cap R=(0)$ and $P$ does not contain $\theta$. By [21, Theorem 4.3] as $R$ is $\alpha$-prime and $\alpha$ has infinite order, every non-zero prime ideal of $S$ which intersects $R$ in (0) contains $\theta$, so $P=(0)$.

Remark 4.4 For use in Sect. 5, we record here a further property of the simple module $V$ defined in Proposition 4.3. Retain the notation of the proposition, and define $\mathcal{A}$ to be the multiplicative subsemigroup of $R \backslash\{0\}$ generated by $\left\{\alpha^{i}(a): i \in \mathbb{Z}\right\}$. Then $\mathcal{A}$ consists of regular elements of $S$, and is easily seen to form an Ore set in that ring. We claim that

$V$ is an $\mathcal{A}$-torsion free simple right $S$-module.

By the proposition, $V$ is simple, so it is either torsion or torsion free. Since $a$ is a regular element of $S$, for all $h \in S \backslash\{0\}$, the degree in $\theta$ of $(1-a \theta) h$ is one more than the degree of $h$. Hence $(1-a \theta) S \cap R=0$. Therefore $1+(1-a \theta) S \in V$ is not $\mathcal{A}$-torsion, and so $V$ is $\mathcal{A}$-torsion free.

\subsection{Reduction to the case where $R$ is a domain}

To achieve the reduction as in the title of the subsection we need the following lemma:

Lemma 4.5 Let $R$ be a commutative Noetherian ring, let $\alpha \in A u t(R)$ and set $S=R[\theta ; \alpha]$. Suppose that $S=R[\theta ; \alpha]$ is prime. Then: 
(a) $R$ is $\alpha$-prime, and so there exist $n \in \mathbb{N}$ and a prime ideal $Q$ of $R$ such that $\left\{Q, \alpha(Q), \ldots, \alpha^{n-1}(Q)\right\}$ is the set of minimal primes of $R$, with $\cap_{i=0}^{n-1} \alpha^{i}(Q)=(0)$.

(b) In the setting of $(a)$, if $S$ is primitive then $(R / Q)\left[\theta^{n} ; \alpha^{n}\right]$ is primitive.

Proof (a) This is clear from Lemma 2.3(c),(d).

(b) With the notation of (a), let $T:=R\left[\theta^{n} ; \alpha^{n}\right]$. Notice that $Q T$ and its $\alpha$-conjugates are the minimal prime ideals of $T$. Suppose that $V$ is a faithful simple right $S$-module. By [12, Theorem 4] $V$ has a (finite) composition series as a $T$-module,

$$
0=V_{0} \subset V_{1} \subset \cdots \subset V_{t}=V .
$$

Set $P_{i}:=\operatorname{Ann}_{T}\left(V_{i} / V_{i-1}\right)$, for $i=1, \ldots, t$, so that

$$
V\left(P_{t} P_{t-1} \ldots P_{1}\right)=0 \text {. }
$$

Since $V$ is by hypothesis a faithful $S$-module, $P_{t} P_{t-1} \ldots P_{1}=(0)$. Hence there exists $j, 1 \leq j \leq t$, with $P_{j} \subseteq Q T$, and the minimality of the prime ideal $Q T$ of $T$ ensures that $P_{j}=Q T$.

That is, $V_{j} / V_{j-1}$ is a faithful simple $T / Q T$-module, as required.

The above lemma, with an equivalence in (b), can be found as [29, Corollary 2.2] with a different proof.

Lemma 4.6 Let $R$ be a commutative Noetherian ring, $\alpha$ an automorphism of $R$, and $S=$ $R[\theta ; \alpha]$.

(a) Let $n \geq 1$ and let $T=R\left[\theta^{n} ; \alpha^{n}\right]$. Then $S$ satisfies $(\diamond)$ if and only if $T$ satisfies $(\diamond)$.

(b) Suppose that $R$ is $\alpha$-prime, with $Q$ and $n$ chosen as in Lemma 4.5(a). If $S$ satisfies $(\diamond)$ so does $T / Q T \simeq(R / Q)\left[\theta^{n} ; \alpha^{n}\right]$.

Proof (a) This follows immediately from Proposition 2.2, since $S$ as a $T$-module is free with a basis formed by the normal elements $1, \theta, \theta^{2}, \ldots, \theta^{n-1}$.

(b) Clear from (a) and Proposition 2.2(c).

\section{$4.3(\diamond)$ When $R$ is an $\alpha$-simple domain}

To investigate which primitive skew polynomial rings over a commutative Noetherian domain $R$ satisfy $(\diamond)$, we first consider the case when $R$ is $\alpha$-simple. We preface the proposition with three lemmas needed for its proof.

Lemma 4.7 Let $\alpha$ be an automorphism of the commutative ring $R$, and suppose that the $\alpha$-orbit of every non-zero prime ideal of $R$ is infinite. Let $\mathcal{P}=\left\{P_{1}, \ldots, P_{t}\right\}$ be a finite set of non-zero prime ideals of $R$. Then there exists a positive integer $n$ such that, for all $i=1, \ldots, t$ and for all $j \in \mathbb{Z} \backslash\{0\}$,

$$
\alpha^{j n}\left(P_{i}\right) \notin \mathcal{P}
$$

Proof For each $i$, there is a positive integer $n_{i}$ such that $\left\{\alpha^{n_{i} t}\left(P_{i}\right): t \in \mathbb{Z}\right\} \cap \mathcal{P}=\left\{P_{i}\right\}$. Thus $n:=\prod_{i} n_{i}$ is as required.

Lemma 4.8 Let $\alpha$ be an automorphism of the commutative ring $R$, let $S=R[\theta ; \alpha]$, and let $\rho$ be a non-zero non-unit of $R$. Then $S / \rho S$ is not an Artinian right $S$-module. 
Proof We claim that the chain

$$
\theta S+\rho S \supseteq \theta^{2} S+\rho S \supseteq \cdots \supseteq \theta^{m} S+\rho S \supseteq \cdots,
$$

of right ideals of $S$ is strictly descending. In order to prove this, suppose that $m \geq 0$ and $\theta^{m} \in \theta^{m+1} S+\rho S=S \theta^{m+1}+\rho S$, say $\theta^{m}=p \theta^{m+1}+\rho q$ for $p, q \in S$. Then $\rho q \in \rho S \theta^{m}$. This implies that $1 \in S \theta+\rho S$, contradicting the fact that $\rho$ is not invertible.

Lemma 4.9 Let $\alpha$ be an automorphism of the ring $R, S=R[\theta ; \alpha]$, and let $\rho$ be a regular non-unit of $R$. Let $\left\{r_{\lambda}: \lambda \in \Lambda\right\}$ be a set of coset representatives for $\rho R$ in $R$. Then every element $m$ of $M:=S / \rho(1-\theta) S$ has a unique expression

$$
m=\sum_{i=\ell}^{k} r_{\lambda_{i}} \theta^{i}+\rho b+\rho(1-\theta) S,
$$

where $b \in R$ and either $(i)$ the part of the expression under the summation symbol is 0 , or else (ii) $\ell \leq k \in \mathbb{Z}_{\geq 0}$ with $r_{\lambda_{\ell}}, r_{\lambda_{k}} \neq 0$.

Proof Since $S=R \oplus(1-\theta) S$ as right $R$-modules and $S \cong \rho S$ as right $S$-modules, the desired expression using coset representatives $\{\rho b: b \in R\}$ for the elements of the submodule $\rho S / \rho(1-\theta) S$ of $M$ is clear. Representation of elements of $M$ by the listed elements follows, since $S / \rho S \cong R / \rho R \otimes_{R} S$ as right $S$-modules.

Proposition 4.10 Let $R$ be a commutative Noetherian domain which is not a field, $\alpha$ an automorphism of $R$ and suppose that $R$ is $\alpha$-simple. Then $S=R[\theta ; \alpha]$ does not satisfy $(\diamond)$. Moreover, there is $n \in \mathbb{N}$ such that $S /\left(1-\theta^{n}\right) S$ is a finite length $S$-module whose injective hull is not locally Artinian.

Proof Let $\rho \in R$ be a non-zero non-unit of $R$, and let $\mathcal{P}=\left\{P_{1}, \ldots, P_{t}\right\}$ be the set of annihilator primes in $R$ of the $R$-module $R / \rho R$. Thus $\rho R \subseteq P_{i}$ for all $i=1, \ldots, t$, and the inverse image in $R$ of every minimal prime of $R / \rho R$ is in $\mathcal{P}$, but there may also be further primes in $\mathcal{P}$. We divide the proof in two cases.

Case 1.

$$
\alpha^{j}\left(P_{i}\right) \notin \mathcal{P} \quad \text { for all } j \in \mathbb{Z} \backslash\{0\}, 1 \leq i \leq t .
$$

Since $R$ is commutative and $\alpha$-simple, $S /(1-\theta) S$ is a simple $S$-module by Lemma 2.4(b). That is, $V:=\rho S / \rho(1-\theta) S$ is a simple submodule of $M:=S / \rho(1-\theta) S$. By Lemma 4.8, $M$ is not an Artinian $S$-module. We shall prove that

$$
V \text { is an essential submodule of } M \text {; }
$$

that is, the thesis holds for $n=1$ in this case.

Let $p$ be a non-zero element of $M$ and apply Lemma 4.9 to write

$$
p=\sum_{i=l}^{k} r_{\lambda_{i}} \theta^{i}+\rho b+\rho(1-\theta) S
$$

for unique $b \in R$ and coset representatives $r_{\lambda_{i}}$ for $\rho R$ in $R$. Here, as in Lemma 4.9, either the summation part of the expression for $p$ is 0 , so that $p \in V$, or we fix $\ell$ and $k$ so that $r_{\lambda_{\ell}}$ and $r_{\lambda_{k}}$ are non-zero. In the former case we define the length $\ell(p)$ of $p$ to be 0 , and in the latter case we define the number of $i$ for which $r_{\lambda_{i}}$ is non-zero to be the length $\ell(p)$ of $p$. The strategy of the proof is to show that, if $\ell(p)>0$, then a non-zero element of strictly shorter length can be found in the submodule $p S$ of $M$. 
Sublemma 1 If $\ell(p)>1$, then $p S$ contains a non-zero element $\widehat{p}$ with $0<\ell(\widehat{p})<\ell(p)$.

Proof of Sublemma 1 Amongst those terms in the expression (6) for $p$ with $r_{\lambda_{i}} \neq 0$, choose $r_{\lambda_{j}}$ such that $r_{\lambda_{j}} R+\rho R / \rho R$ has a maximal annihilator ideal $Q$ which has minimal height amongst all the maximal annihilators in $R$ of the non-zero $R$ modules in the list $\left\{r_{\lambda_{i}} R+\right.$ $\rho R / \rho R: \ell \leq i \leq k\}$. Let $r \in R$ be such that

$$
\operatorname{Ann}_{R}(X)=Q
$$

for every non-zero $R$-submodule $X$ of $r_{\lambda_{j}} r R+\rho R / \rho R$. Now multiply $p$ by $\alpha^{-j}(r)$, to get

$$
\begin{aligned}
p_{1} & :=p \alpha^{-j}(r) \\
& =r_{\lambda_{j}} r \theta^{j}+\sum_{i \neq j} r_{\lambda_{i}} \alpha^{i-j}(r) \theta^{i}+\rho b \alpha^{-j}(r)+\rho(1-\theta) S .
\end{aligned}
$$

Observe that, since we are assuming $\ell(p)>1$, the summation over $i$ in the above expression is not empty. If one or more of the terms appearing under the summation symbol features $r_{\lambda_{i}} \alpha^{i-j}(r) \in \rho R$, then the proof of the sublemma is complete, taking $\widehat{p}:=p_{1}$. Suppose then that this is not the case, and choose $w, \ell \leq w \leq k, w \neq j$, such that $r_{\lambda_{w}} \alpha^{w-j}(r) R$ has a maximal annihilator ideal $J$ which is maximal amongst annihilator ideals of non-zero elements of the $R$-modules in the collection $\left\{r_{\lambda_{i}} \alpha^{i-j}(r) R+\rho R / \rho R: i \neq j\right\}$. Choose $\widehat{r} \in R$ such that

$$
\operatorname{Ann}_{R}\left(r_{\lambda_{w}} \alpha^{w-j}(r) \widehat{r}+\rho R\right)=J .
$$

Now multiply $p_{1}$ by $\alpha^{-w}(\widehat{r})$ to get

$$
\begin{aligned}
p_{2}:= & p_{1} \alpha^{-w}(\widehat{r}) \\
= & r_{\lambda_{j}} r \alpha^{j-w}(\widehat{r}) \theta^{j}+\sum_{i \neq j, w} r_{i} \alpha^{i-j}(r) \alpha^{i-w}(\widehat{r}) \theta^{i} \\
& +r_{\lambda_{w}} \alpha^{w-j}(r) \widehat{r} \theta^{w}+\rho b \alpha^{-j}(r) \alpha^{-w}(\widehat{r})+\rho(1-\theta) S .
\end{aligned}
$$

If $r_{\lambda_{j}} r \alpha^{j-w}(\widehat{r}) \in \rho R$ then the sublemma is proved. So, suppose on the other hand that

$$
r_{\lambda_{j}} r \alpha^{j-w}(\widehat{r}) \notin \rho R
$$

Observe that $\alpha^{j-w}(J)$ cannot be strictly contained in $Q$, since

$$
\operatorname{height}(Q) \leq \operatorname{height}(J)=\operatorname{height}\left(\alpha^{j-w}(J)\right),
$$

thanks to our choice of $Q$. Moreover, thanks to (4), we cannot have $\alpha^{j-w}(J)=Q$. Thus it is possible to choose $x \in J$ with $\alpha^{j-w}(x) \notin Q$. Then

$$
\widehat{p}:=p_{2} \alpha^{-w}(x) \in p S \subseteq M
$$

satisfies

$$
0<\ell(\widehat{p})<\ell(p)
$$

and the sublemma is proved.

Sublemma 2 Let $p \in M$ be written in normal form (6), with $\ell(p)=1$ - that is, $\ell=k$ and $r_{\lambda_{k}} \neq 0$. Then there exists $u \in R$ such that $0 \neq p u \in V$. 
Proof of Sublemma 2 Simplifying notation slightly, let $p$ have coset representative

$$
r_{i} \theta^{i}+\rho b \in S \backslash \rho(1-\theta) S,
$$

where $0 \neq r_{i} \in R \backslash \rho R, b \in R$, and $i \geq 0$. We have to show that there exists $u \in R$ such that

$$
\left(r_{i} \theta^{i}+\rho b\right) u \in \rho S \backslash \rho(1-\theta) S .
$$

We prove that (10) is true with $u=\alpha^{-i}(\rho)$. For $\left(r_{i} \theta^{i}+\rho b\right) \alpha^{-i}(\rho)$ clearly lies in $\rho S$. On the other hand,

$$
\begin{aligned}
\left(r_{i} \theta^{i}\right. & +\rho b) \alpha^{-i}(\rho) \in \rho(1-\theta) S \\
& \Leftrightarrow \rho r_{i} \theta^{i}+\rho b \alpha^{-i}(\rho) \in \rho(1-\theta) S \\
& \Leftrightarrow r_{i} \theta^{i}+b \alpha^{-i}(\rho) \in(1-\theta) S \\
& \Leftrightarrow\left(\theta^{i}-1\right) \alpha^{-i}\left(r_{i}\right)+\alpha^{-i}\left(r_{i}\right)+b \alpha^{-i}(\rho) \in(1-\theta) S \\
& \Leftrightarrow \alpha^{-i}\left(r_{i}\right)=-b \alpha^{-i}(\rho) \\
& \Leftrightarrow r_{i}=-\rho \alpha^{i}(b),
\end{aligned}
$$

where the penultimate equivalence follows from Lemma 2.4(a). But $r_{i}=-\rho \alpha^{i}(b)$ is a contradiction to the initial hypothesis that $r_{i} \notin \rho R$, so that (10) is true and Sublemma 2 is proved.

Thus (5) follows at once from the two sublemmas, and $V=S /(1-\theta) S$ is a simple $S$-module with an injective hull that is not locally Artinian.

Case 2. Assume that (4) does not hold. Note that if $R$ is $\alpha$-simple, then $R$ is also $\alpha^{m}$-simple for any $m \in \mathbb{N}$. Indeed if $I$ is $\alpha^{m}$-stable proper ideal of $R$, then $I \alpha(I) \ldots \alpha^{m-1}(I)=0$ as it is an $\alpha$-stable proper ideal, and so $I=(0)$ since $R$ is a domain. Hence also every non-zero prime ideal has an infinite $\alpha^{m}$-orbit. Choose $n \in \mathbb{N}$ such that the conclusion of Lemma 4.7 holds for $\alpha$ and $\mathcal{P}$. By the above we can apply Case 1 to $S^{\prime}=R\left[\theta^{n} ; \alpha^{n}\right]$ and obtain that $V=S^{\prime} /\left(1-\theta^{n}\right) S^{\prime}$ is a simple $S^{\prime}$-module with an injective hull that is not locally Artinian.

As an $S^{\prime}$-module, $S$ is free with a basis formed by normal elements, $1, \theta, \ldots, \theta^{n-1}$. The $S$-module $V \otimes_{S^{\prime}} S \simeq S /\left(1-\theta^{n}\right) S$ is, as an $S^{\prime}$-module, isomorphic to

$$
\bigoplus_{i=0}^{n-1} V^{\alpha^{i}}
$$

where $V^{\alpha^{i}}$ is the $S^{\prime}$-module defined by taking $V$ as the underlying Abelian group and setting $v \cdot s=v \alpha^{i}(s)$, where $\alpha^{i}$ is the automorphism of $S^{\prime}$ induced by $\alpha^{i}$. Since $V$ is simple as $S^{\prime}$-module, so is each $V^{\alpha^{i}}$. Hence $V \otimes_{S^{\prime}} S$ is a finite length $S^{\prime}$-module. Therefore $V \otimes_{S^{\prime}} S$ is a finite length $S$-module.

Since $S$ is free as an $S^{\prime}$-module, $V \otimes_{S^{\prime}} S \leq E_{S^{\prime}}(V) \otimes_{S^{\prime}} S$. Write $\leq_{e}$ for "is an essential submodule of", and observe that $V \otimes_{S^{\prime}} S \leq_{e} E_{S^{\prime}}(V) \otimes_{S^{\prime}} S$ as $S^{\prime}$-modules, and hence $a$ fortiori as $S$-modules. So

$$
E_{S^{\prime}}(V) \otimes_{S^{\prime}} S \leq_{e} E_{S}\left(V \otimes_{S^{\prime}} S\right) .
$$

Since $E_{1}:=E_{S^{\prime}}(V) \otimes_{S^{\prime}} S$ is contained in $E_{S}(V \otimes S)$ and is not locally Artinian, there is a finitely generated $S^{\prime}$-submodule $M$ of $E_{1}$ which is not $S^{\prime}$-Artinian. The finitely generated $S$-submodule $M S$ of $E_{1}$. Then $M S$ is finitely generated also as an $S^{\prime}$-module, and so, by [12, 
Theorem 4] if $M S$ were $S$-Artinian, then $M S$ would be $S^{\prime}$-Artinian. This is a contradiction, since $M \leq M S$ as $S^{\prime}$-submodule. So $M S$ is not $S$-Artinian and $E_{S}(V \otimes S)$ is not a locally Artinian $S$-module, as claimed.

In fact it can be shown, using an argument based on [44, Exercise 31 on page 112] that $E_{S^{\prime}}(V) \otimes_{S^{\prime}} S=E_{S}(V \otimes S)$ but we do not give details here since we do not need that fact.

\section{Necessary and sufficient conditions for $(\diamond)$}

In handling primitive skew polynomial rings our approach is to strengthen the $\alpha$-special property, guaranteed by Theorem 4.2 for such a primitive ring, to the stronger $\alpha$-simple condition. This can be achieved by localising at the smallest $\alpha$-stable Ore set $\mathcal{A}$ of $S$ containing the $\alpha$-special element. However, to apply Proposition 4.10 after this localisation, we need to exclude the possibility that $R \mathcal{A}^{-1}$ is a field. To achieve this, we need to assume that $R$ is "sufficiently big" in terms both of the height and the "width" of its lattice of prime ideals, and that it contains an uncountable field. The technicalities for this are provided by the lemmas of Sect. 5.1. The key results are then Proposition 5.3 and Theorem 5.4.

\subsection{Localisation lemmas}

Lemma 5.1 Let $T$ be a ring and $\mathcal{A}$ a multiplicatively closed Ore subset of regular elements of $T$. If there exists a right $T$-module $V$ such that $E_{T \mathcal{A}^{-1}}\left(V \otimes_{T} T \mathcal{A}^{-1}\right)$ is not locally Artinian as a $T \mathcal{A}^{-1}$-module, then $E_{T}(V / \tau(V))$ is not locally Artinian, where $\tau(V)$ denotes the $\mathcal{A}$-torsion submodule of $V$.

Proof Suppose that $E_{T \mathcal{A}^{-1}}\left(V \otimes T \mathcal{A}^{-1}\right)$ is not locally Artinian as a $T \mathcal{A}^{-1}$-module. In particular $V$ is not $\mathcal{A}$-torsion. Let $\tau(V)$ be the $\mathcal{A}$-torsion submodule of $V$, then $\frac{V}{\tau(V)} \otimes T \mathcal{A}^{-1} \simeq$ $V \otimes T \mathcal{A}^{-1}$. Thus replacing $V$ by $V / \tau(V)$ we may assume that $\tau(V)=0$.

It is easy to check that $E_{T \mathcal{A}^{-1}}\left(V \otimes_{T} T \mathcal{A}^{-1}\right)$ is an essential extension of $V$ as a $T$-module. In particular this implies that

$$
E_{T \mathcal{A}^{-1}}\left(V \otimes_{T} T \mathcal{A}^{-1}\right) \leq E_{T}(V) .
$$

Now let $e_{1}, \ldots, e_{n} \in E_{T \mathcal{A}^{-1}}\left(V \otimes_{T} T \mathcal{A}^{-1}\right)$ be such that $W:=\sum_{j=1}^{m} e_{j} T \mathcal{A}^{-1}$ contains an infinite descending chain

$$
\cdots \subset W_{i+1} \subset W_{i} \subset \cdots \subset W_{2} \subset W_{1}=W
$$

of $T \mathcal{A}^{-1}$-submodules of $W$. For all $i, W_{i}=\left(W_{i} \cap \sum_{j=1}^{m} e_{j} T\right) T \mathcal{A}^{-1}$, so (11) yields an infinite strictly descending chain of $T$-submodules of $\sum_{j=1}^{m} e_{j} T$, as required.

The example (already featuring in Sect. 4) of $R=k[[X]]$ and $\mathcal{A}=\left\{X^{i}: i \geq 0\right\}$ should be borne in mind in conjunction with the next lemma.

Lemma 5.2 Let $R$ be a commutative Noetherian domain which is an algebra over an uncountable field, but is not itself a field. Suppose that there exists a countable multiplicatively closed subset $\mathcal{A}$ of $R \backslash\{0\}$ such that $R \mathcal{A}^{-1}$ is the quotient field of $R$. Then $R$ has Krull dimension 1 and $\operatorname{Spec}(R)$ is countable. 
Proof Assume $R$ and $\mathcal{A}$ are as above. By [41, Proposition 2.5] every Noetherian ring containing an uncountable field has the countable prime avoidance property. It follows from [27, Theorem 3.8] that $\operatorname{Spec}(R)$ is countable and that each non-zero prime ideal is maximal.

\section{$5.2(\diamond)$ when $S$ is primitive}

Recall that the multiplicatively closed $\alpha$-stable Ore subset $\mathcal{A}$ of $S$ generated by an $\alpha$-special element $a$ of $R$ was defined in Remark 4.4.

Proposition 5.3 Let $R$ be a commutative Noetherian domain, $\alpha$ an automorphism of $R$. Suppose that $S=R[\theta ; \alpha]$ is primitive, with $\alpha$-special element a and associated Ore subset $\mathcal{A}$. Suppose that $R \mathcal{A}^{-1}$ is not a field. Then $S$ does not satisfy $(\diamond)$.

Proof The existence of the element $a$ is guaranteed by Theorem 4.2. Note that $R \mathcal{A}^{-1}$ is $\alpha$-simple and $S \mathcal{A}^{-1}=R \mathcal{A}^{-1}[\theta ; \alpha]=R \mathcal{A}^{-1}[a \theta ; \alpha]$. By Proposition 4.10 there is $n \in \mathbb{N}$ such that

$$
E_{S \mathcal{A}^{-1}}\left(S \mathcal{A}^{-1} /\left(1-(a \theta)^{n}\right) S \mathcal{A}^{-1}\right)
$$

is not locally Artinian.

Set $S^{\prime}=R\left[\theta^{n} ; \alpha^{n}\right]$. Since for any $\alpha^{n}$-stable ideal $I$ of $R$, the ideal $I \alpha(I) \ldots \alpha^{n-1}(I)$ is $\alpha$-stable, it is clear that $R$ is $\alpha^{n}$-special with the special element $b=a \alpha(a) \alpha^{2}(a) \ldots \alpha^{n-1}(a)$. Thus $S^{\prime}$ is primitive and by Proposition 4.3, $V=S^{\prime} /\left(1-b \theta^{n}\right) S^{\prime}=S^{\prime} /\left(1-(a \theta)^{n}\right) S^{\prime}$ is simple as a $S^{\prime}$-module since $b \theta^{n}=(a \theta)^{n}$.

By similar arguments to the ones at the end of the proof of Proposition 4.10, $W:=V \otimes S=$ $S /\left(1-(a \theta)^{n}\right) S$ is of finite length as $S$-module. By Lemma 5.1 the injective hull of the finite length module $W / \tau(W)$ is not locally Artinian and the result follows.

We can now give necessary and sufficient conditions for $S=R[\theta ; \alpha]$ to satisfy $(\diamond)$ when $S$ is primitive. Unfortunately, however, these two conditions do not together cover all possibilities.

Theorem 5.4 Let $R$ be a commutative Noetherian ring, $\alpha$ an automorphism of $R$, and let $S=R[\theta ; \alpha]$. Suppose that $S$ is primitive.

(a) If $R$ has Krull dimension 0 then $S$ satisfies $(\diamond)$.

(b) Suppose that $R$ contains an uncountable field. Suppose also that either $R$ has Krull dimension at least 2 , or $\operatorname{Spec}(R)$ is uncountable. Then $S$ does not satisfy $(\diamond)$.

Proof (a) When $R$ has Krull dimension $0, S$ has Krull dimension 1 by [16, Theorem 15.19]. Since $S$ is a prime Noetherian ring of Krull dimension 1, $(\diamond)$ follows easily (see for instance [32, Proposition 5.5]).

(b) Suppose that $R$ contains an uncountable field, and that $R$ has Krull dimension 2 or more, or has uncountably many prime ideals. Let $Q$ be a minimal prime ideal of $R$, let $n \in \mathbb{N}$ be such that the minimal primes of $R$ are $\left\{\alpha^{i}(Q): 0 \leq i \leq n-1\right\}$, and set $T:=R\left[\theta^{n} ; \alpha^{n}\right]$. By Lemma 4.5(b) $T / Q T$ is also primitive, and by Lemma 4.6(b) it is enough to show that $T / Q T$ does not satisfy $(\diamond)$. In other words, in proving (b), we can pass to $(R / Q)\left[\theta^{n} ; \alpha^{n}\right]$, observing that $R / Q$ will like $R$ contain an uncountable field, and have Krull dimension at least 2 or have uncountably many prime ideals, just as $R$ does. That is, we may assume additionally that $R$ is a domain in proving (b).

Let $a$ be the $\alpha$-special element of $R$ guaranteed by Theorem 4.2, with $\mathcal{A}$ the $\alpha$-stable Ore set it generates. By Lemma $5.2 R \mathcal{A}^{-1}$ cannot be the quotient field of $R$. Proposition 5.3 now implies that $S$ does not satisfy $(\diamond)$. 
It is straightforward to show that in Theorem 5.4 under the hypothesis (a), the ring $R$ is a finite direct sum of isomorphic fields.

\section{Property $(\diamond)$ when $R$ is affine}

The gap between parts (a) and (b) of Theorem 5.4 can be closed so as to determine completely the occurrence of $(\diamond)$ for $S=R[\theta ; \alpha]$, provided $R$ contains an uncountable field and has no $\alpha$-invariant factors of Krull dimension 1 whose spectrum is countable. In particular, this allows us to completely settle the matter when $R$ is an affine algebra over an uncountable field, as follows.

Theorem 6.1 Let $k$ be an uncountable field and $R$ an affine $k$-algebra, and let $\alpha$ be a $k$-algebra automorphism of $R$. Let $S=R[\theta ; \alpha]$. Then the following are equivalent:

(a) S satisfies $(\diamond)$;

(b) all simple $S$-modules are finite dimensional $k$-vector spaces.

Proof $(\mathrm{b}) \Rightarrow$ (a) This follows immediately from Propositions 3.2 and 3.3 and does not require that $k$ is uncountable.

(a) $\Rightarrow$ (b) Suppose that $S$ satisfies $(\diamond)$. Let $V$ be a simple $S$-module and $P=\operatorname{Ann}_{S}(V)$.

If $\theta \in P$, then $P / \theta S$ is a primitive ideal of the commutative affine $k$-algebra $R$, so $V$ is finite dimensional over $k$, thanks to the Nullstellensatz.

Suppose next that $(P \cap R) S \subset P$ and $\theta \notin P$. Then [21, Theorem 4.3] implies that $S /(P \cap R) S$ satisfies a polynomial identity. Since $S /(P \cap R) S$ is also by hypothesis and construction a Noetherian affine $k$-algebra, $V$ is finite dimensional over $k$ by Kaplansky's theorem, [4, Theorem I.13.3].

Suppose finally that $(P \cap R) S=P$ and let $Q$ as usual denote a minimal prime over $P \cap R$ in $R$. If $R / P \cap R$ has Krull dimension 0, it is a finite direct sum of copies of the field $R / Q:=F$, by Lemma 2.3(d). Hilbert's Nullstellensatz ensures that $F$ is finite dimensional over $k$, so that some finite power, say $n$, of $\alpha$ not only fixes $Q$, but then also induces the identity on $F$. Hence, $S / P$ is a finite (free) module over the commutative $\operatorname{ring}(R / P \cap R)\left[\theta^{n}\right]$, so $S / P$ satisfies a polynomial identity and Kaplansky's theorem applies. Suppose on the other hand that $R / P \cap R$ has Krull dimension at least 1. Then, for example using Lying Over and the Noether normalisation theorem [10, Proposition 4.15 and $\$ 8.2 .1$, Theorem A1], $\operatorname{Spec}(R / Q)$ is uncountable since $k$ is uncountable. By Theorem 5.4(b), this contradicts property $(\diamond)$ for $S$. So no such primitive ideals $P$ can exist in $S$ and (b) follows.

Remark (i) Notice that $(b) \Rightarrow(a)$ of this theorem is valid, with the same proof, for an arbitrary commutative Noetherian $k$-algebra over any field. Indeed, if we change statement (b) to

\section{$\left(b^{\prime}\right) S / \operatorname{Ann}_{S}(V)$ is Artinian for all simple $S$-modules $V$,}

then a small adjustment to the argument confirms that $\left(b^{\prime}\right) \Rightarrow(a)$ is true for all commutative Noetherian coefficient rings $R$.

(ii) Following circulation of an earlier draft of this paper, Jason Bell [2] has explained to us how to remove the hypothesis that $k$ is uncountable from Theorem 6.1, at least when $k$ has characteristic 0. The key point is to obtain a replacement for Proposition 5.3 in the case where $R$ is $k$-affine and $k$ has characteristic 0 . The substitute result is: 
Proposition 5.3' Let $k$ be a field of characteristic 0 and let $R$ be a commutative $k$-affine domain which is not a field. Let $\alpha$ be a k-algebra automorphism of $R$. Suppose that $S=R[\theta ; \alpha]$ is primitive, with $\alpha$-special element a and associated Ore subset $\mathcal{A}$. Then $R \mathcal{A}^{-1}$ is not a field and hence $S$ does not satisfy $(\diamond)$.

With Proposition 5.3' to hand, one can follow the proof of Theorem 6.1 (a) $\Rightarrow(\mathrm{b})$, invoking 5.3' to handle the crucial case where $P=(P \cap R) S$ with $Q$ a minimal prime over $P \cap R$, by deducing that then $R / Q$ is a field.

Here is a brief indication of how to prove Proposition 5.3'. First observe that the automorphism $\alpha$ and the $\alpha$-special element $a$ are defined in a finitely generated field extension $K$ of $\mathbb{Q}$. We can then work over a finitely generated $\mathbb{Z}$-algebra $T$ whose field of fractions is $K$. One can specialise modulo the maximal ideals of $T$, so to $\mathbb{F}_{q}$-algebras for prime powers $q$, and apply model-theoretic work of Hrushovski, [20, Corollary 1.2], using the $\mathbb{F}_{q}$-Frobenius to see that that the $\alpha$-special configuration with the localisation at the image of $\mathcal{A}$ being a field is impossible. This conclusion then lifts back to $R$, proving 5.3'.

Let $k$ be any field, let $R$ be a commutative affine $k$-algebra and let $\alpha$ be a $k$-algebra automorphism of $R$. In the light of Theorem 6.1 it is natural to ask exactly what conditions on $R$ and $\alpha$ are required for (b) of Theorem 6.1 to hold. This appears to be quite a subtle question, which we discuss further in Sect. 7. First, we give here an important special case in which a complete answer is available:

Proposition 6.2 Let $k$ be a field, $t$ a positive integer, $V$ a vector space over $k$ with basis $\left\{x_{1}, \ldots, x_{t}\right\}$ and $\alpha \in \mathrm{GL}(t, k)$ an automorphism of $V$. Let $R=k\left[x_{1}, \ldots, x_{t}\right]$, so that $\alpha$ induces a $k$-algebra automorphism of $R$, also denoted by $\alpha$. Then $S:=R[\theta ; \alpha]$ satisfies $(\diamond)$ if and only if the order $|\alpha|$ of the automorphism $\alpha$ is finite.

Proof Suppose $|\alpha|=n<\infty$. Since the commutative Noetherian ring $R\left[\theta^{n}\right]$ satisfies $(\diamond)$, Lemma 4.6(a) implies that $\mathrm{S}$ satisfies $(\diamond)$.

For the converse, suppose that $S$ satisfies $(\diamond)$. Let $L$ be a finite extension field of $k$ such that the Jordan Normal Form of $\alpha$ exists in $G L(t, L)$. By Proposition $2.2 L \otimes_{k} S$ satisfies $(\diamond)$, so we may replace $R$ by $\widehat{R}=L\left[x_{1}, \ldots, x_{t}\right]$ and $S$ by $\widehat{S}:=\widehat{R}[\theta ; \alpha]$ in proving that $|\alpha|<\infty$. Changing the basis of $V$ as necessary, we can assume that $\alpha$ has Jordan Normal Form with respect to the ordered basis $\left\{x_{1}, \ldots, x_{t}\right\}$. We show first that

every generalised eigenvalue of $\alpha$ is a root of unity in $L$.

Suppose that (12) is false, so there exists $i$ such that the generalised eigenvalue $a_{i}$ of $x_{i}$ is not a root of 1 . Choose $i$ and $j \leq i$ so that $\left\{x_{j}, \ldots, x_{i}\right\}$ is a basis of the Jordan block of $\alpha$ for the eigenvalue $a_{i}$. Then it is clear that

$$
P:=\sum_{\ell \neq i} x_{\ell} \widehat{R}
$$

is an $\alpha$-invariant ideal of $\widehat{R}$. Thus $P \widehat{S} \triangleleft \widehat{S}$, with

$$
\widehat{S} / P \widehat{S} \cong(\widehat{R} / P)[\theta ; \bar{\alpha}]=L\left[x_{i}\right][\theta ; \bar{\alpha}],
$$

slightly abusing notation by writing $x_{i}$ for the image of that element of $\widehat{R}$ in $\widehat{R} / P$, and where $\bar{\alpha}$ is the $L$-algebra automorphism of $L\left[x_{i}\right]$ defined by $\bar{\alpha}\left(x_{i}\right)=a_{i} x_{i}$. Thus $\widehat{S} / P \widehat{S}$ is a quantum plane with parameter $a_{i}$ and does not satisfy $(\diamond)$ by [8, Theorem 3.1]. This contradicts the hypothesis that $S$ and therefore $\widehat{S}$ satisfy $(\diamond)$, so (12) is proved. (This can also be seen with the help of Proposition 5.3. Indeed one checks easily that $x_{i}$ is an $\bar{\alpha}$-special element of $\widehat{R} / P$, 
with corresponding $\bar{\alpha}$-stable Ore set $\mathcal{A}$ in $\widehat{R} / P$ equal to $\left\{a_{i}^{m} x_{i}^{n}: m \in \mathbb{Z}, n \in \mathbb{N}\right\}$. Hence $\widehat{S} / P \widehat{S}$ is primitive and since $(\widehat{R} / P) \mathcal{A}^{-1}$ is not a field, we conclude from Proposition 5.3 that $\widehat{S} / P \widehat{S}$ does not satisfy $(\diamond)$.)

Notice also that this completes the proof of the proposition in the case where $k$, or equivalently $L$, has positive characteristic, since then the matrix of $\alpha$ lies in $G L(t, E)$ for some finite field $E$.

Now suppose $L$ has characteristic 0 , and that $\alpha$ is not diagonalisable, so that there is a Jordan block of $\alpha$ of cardinality greater than 1 . Let $n$ be the least common multiple of the orders of the generalised eigenvalues of $\alpha$. Replacing $\widehat{S}$ by $\widehat{R}\left[\theta^{n} ; \alpha^{n}\right]$, using Lemma 4.6(a), we may assume that the only generalised eigenvalue of $\alpha$ is 1 . We aim for a contradiction to the hypothesis that $\widehat{S}$ satisfies $(\diamond)$. Rearranging the order of the basis vectors of $V$ if need be, we can assume that there is a block of $\alpha$ with basis $\left\{x_{m}, \ldots, x_{t}\right\}$, for some $m<t$. Consider now the $\alpha$-invariant ideal of $\widehat{R}$,

$$
I:=\sum_{\ell=1}^{t-2} x_{\ell} \widehat{R}+\left(x_{t-1}-1\right) \widehat{R} .
$$

Passing to $\widehat{S} / I \widehat{S} \cong(\widehat{R} / I)[\theta ; \alpha] \cong L\left[x_{t}\right][\theta ; \bar{\alpha}]$, we may assume that

$$
\bar{\alpha}: x_{t} \mapsto x_{t}+1 \text {. }
$$

One readily checks that $\widehat{S} / I \widehat{S}$ is isomorphic to the enveloping algebra of the 2-dimensional solvable non-abelian Lie algebra over a field of characteristic 0 , so it does not satisfy $(\diamond)$, by [33]; alternatively, since $L$ has characteristic $0, \widehat{R} / I$ is $\alpha$-simple and one can appeal directly to Proposition 4.10 to get a contradiction to property $(\diamond)$ for $\widehat{S}$. Therefore, $\alpha$ is diagonalisable; coupling this with (12), the proposition is proved.

\section{Examples and discussion}

We discuss in this section property $(\diamond)$ for $S=R[\theta ; \alpha]$ when the base ring $R$ is an integral domain and an affine $k$-algebra over a field $k$, and $\alpha$ is a $k$-algebra automorphism of $R$. In the light of Theorem 6.1 and Proposition 6.2, this amounts to examining the interconnections between the following four hypotheses:

$(\bullet) S$ satisfies $(\diamond)$.

$(\bullet)$ Every simple $S$-module has finite dimension over $k$.

(•) $S$ satisfies a polynomial identity.

(•) $\alpha$ has finite order.

We have seen in Sect. 6 that the first two of these are equivalent, that the third and fourth are equivalent, by [9], and that the third implies the second, by Kaplansky's theorem, [4, Theorem I.13.3]. Moreover all four are equivalent when $\alpha$ is a linear automorphism of a polynomial algebra, by Proposition 6.2.

We first consider, in Sects. 7.1 and 7.2, the application of Theorem 6.1 to the algebras $S_{k, n, \alpha}:=k\left[x_{1}, \ldots, x_{n}\right][\theta ; \alpha]$, where $k$ is a field, $n$ is a positive integer, and $\alpha$ is as a $k$ algebra automorphism of $k\left[x_{1}, \ldots, x_{n}\right]$. Note that if $\xi \in A=\operatorname{Aut}\left(k\left[x_{1}, \ldots, x_{n}\right]\right)$, the group of $k$-algebra automorphisms of $R=k\left[x_{1}, \ldots, x_{n}\right]$, then $\xi$ extends to an isomorphism from $R[\theta ; \alpha]$ to $R\left[\theta ; \xi \alpha \xi^{-1}\right]$. Thus, when convenient, we may replace $\alpha$ by its conjugate. Here, we will only consider $n=1$ and $n=2$, but even for these small values of $n$ the situation turns out to be surprisingly delicate. The representation theory of $S_{k, n, \alpha}$ likely has close interactions 
with the dynamical properties of $\alpha$, as studied for example in [11] and subsequent works; see for instance [37]. Even for the case $n=2$ we are unable to determine whether the first bullet point above is equivalent to the third and fourth.

We then turn in Sect. 7.3 to algebras $S=R[\theta ; \alpha]$ occurring as subalgebras of group algebras $k G$ of torsion-free polycyclic groups $G$, where $k$ is algebraic over a field of $p$ elements, $p$ prime. Here, thanks to deep results on the representation theory of these group algebras, we can easily construct many examples where the first two of the above bullet points hold, but the second two do not.

In the final subsection, Sect. 7.4, we describe an example due to Jordan (cf. [25]), which shows that there is no equivalence of the four bullet points for affine algebras in characteristic 0 .

\section{$7.1(\diamond)$ for $S_{k, 1, \alpha}$}

Here $k$ can be an arbitrary field. As is easy to confirm, the group $A$ of $k$-algebra automorphisms of $k[x]$ consists of the affine automorphisms, mapping $x$ to $\beta x+\gamma$, for $\beta, \gamma \in k$ with $\beta$ non-zero.

Proposition 7.1 With the above notation, the following are equivalent.

(a) $S_{k, 1, \alpha}$ satisfies $(\diamond)$;

(b) every simple $S_{k, 1, \alpha}$-module is finite dimensional over $k$;

(c) $S_{k, 1, \alpha}$ is a finite module over its centre;

(d) $\alpha$ is of finite order, i.e if $\beta \neq 1$ then $\beta$ is a root of unity and if $\beta=1, \gamma=0$ when $k$ has characteristic 0 .

Proof The implications $(\mathrm{d}) \Rightarrow(\mathrm{c}) \Rightarrow(\mathrm{b}) \Rightarrow(\mathrm{a})$ are standard. Indeed suppose (d) holds. Then $\alpha$ is of finite order and by Noether's Theorem [42, Theorem 2.3.1], (c) follows easily. Moreover $S$ satisfies a polynomial identity and by Kaplansky's Theorem [4, Theorem I.13.3] we have that (c) $\Rightarrow(\mathrm{b})$. The implication (b) $\Rightarrow$ (a) follows as in Theorem 6.1.

(a) $\Rightarrow$ (d) Suppose that $S_{k, 1, \alpha}$ satisfies $(\diamond)$. Note that if $\beta \neq 1$ then $\alpha$ is conjugate to $\alpha^{\prime} \in \operatorname{Aut}(k[x])$ such that $\alpha^{\prime}(x)=\beta x$. Thus by Proposition 6.2 (or [8, Theorem 3.1]) it follows that $\beta$ is a root of unity.

If $\beta=1$ and $k$ has characteristic 0 then, by Proposition 4.10, $\gamma=0$.

\section{$7.2(\diamond)$ for $S_{\mathbb{C}, 2, \alpha}$}

Consider here $S_{\mathbb{C}, 2, \alpha}=\mathbb{C}[x, y][\theta ; \alpha]$. Let $A:=\operatorname{Aut}(\mathbb{C}[x, y])$, the group of $\mathbb{C}$-algebra automorphisms of $R:=\mathbb{C}[x, y]$, and let $\alpha \in A$. Following [43, Proposition 1], $\alpha$ is triangular if it is conjugate in $A$ to one of the following types of automorphism:

(i) $x \mapsto \lambda x ; \quad y \mapsto \mu y, \quad \lambda, \mu \in \mathbb{C} \backslash\{0\}$;

(ii) $x \mapsto \lambda x ; \quad y \mapsto y+c, \quad \lambda, c \in \mathbb{C} \backslash\{0\}$;

(iii) $x \mapsto \lambda x+\sum_{\left\{i: \lambda=\mu^{i}\right\}} \eta_{i} y^{i} ; \quad y \mapsto \mu y, \quad \eta_{i} \in \mathbb{C}, \lambda, \mu \in \mathbb{C} \backslash\{0\}$.

As remarked before, throughout the arguments below, we may where convenient replace a hypothesis that $\alpha$ is conjugate to an element of type (j) by the hypothesis that $\alpha$ belongs to type (j). A similar comment applies also to the later proofs in this subsection.

Lemma 7.2 With the notation as above, suppose that $\alpha$ is triangular. Then the following are equivalent: 
(a) $S_{\mathbb{C}, 2, \alpha}$ satisfies $(\diamond)$;

(b) $\alpha$ is conjugate to an automorphism of type ( $i)$, with $\lambda$ and $\mu$ both roots of unity;

(c) $\alpha$ has finite order;

(d) $S_{\mathbb{C}, 2, \alpha}$ is a finite module over its centre;

(e) every simple $S_{\mathbb{C}, 2, \alpha}$-module is a finite dimensional $k$-vector space.

Proof The implication (b) $\Rightarrow(c)$ is clear and $(c) \Rightarrow(d) \Rightarrow(e)$ follow as in the first paragraph of the proof of Proposition 7.1. Finally, (e) $\Rightarrow$ (a) is a special case of (b) $\Rightarrow$ (a) of Theorem 6.1.

To prove (a) $\Rightarrow$ (b) suppose that $S_{\mathbb{C}, 2, \alpha}$ satisfies $(\diamond)$. If $\alpha$ is of type (ii), then $x S_{\mathbb{C}, 2, \alpha}$ is an ideal of $S_{\mathbb{C}, 2, \alpha}$ and $S_{\mathbb{C}, 2, \alpha} / x S_{\mathbb{C}, 2, \alpha}$ is isomorphic to $\mathbb{C}[y][\theta ; \beta]$ where $\beta(y)=y+c$ for $c \neq 0$. By Propositions 7.1 and $2.2(\mathrm{c}), S_{\mathbb{C}, 2, \alpha}$ does not satisfy $(\diamond)$, contradicting our hypothesis.

Suppose alternatively that $\alpha$ is of type (iii) but not type (i). Since $y S_{\mathbb{C}, 2, \alpha}$ is an ideal of $S_{\mathbb{C}, 2, \alpha}$ and $S_{\mathbb{C}, 2, \alpha} / y S_{\mathbb{C}, 2, \alpha}$ is isomorphic to a quantum plane with parameter $\lambda$. By Propositions 7.1 and 2.2 (c), $\lambda$ is a root of unity. Since $S_{\mathbb{C}, 2, \alpha}$ is by hypothesis not type (i), this forces $\mu$ also to be a root of unity. By Proposition 2.2 the subalgebra $\mathbb{C}[x, y]\left[\theta^{n} ; \alpha^{n}\right]$ of $S_{\mathbb{C}, 2, \alpha}$ also satisfies $(\diamond)$, so we may pass to that subalgebra for a suitable choice of $n$, and thus assume that $\mu=1=\lambda$, (but keep the notation $S_{\mathbb{C}, 2, \alpha}=\mathbb{C}[x, y][\theta ; \alpha]$ ). Let $\tau \in \mathbb{C}$ be a root of the polynomial $\sum_{\lambda=\mu^{i}} \eta_{i} y^{i}-1$. Then $(y-\tau) S_{\mathbb{C}, 2, \alpha}$ is an ideal of $S_{\mathbb{C}, 2, \alpha}$, with $S_{\mathbb{C}, 2, \alpha} /(y-\tau) S_{\mathbb{C}, 2, \alpha}$ isomorphic to $\mathbb{C}[x][\theta ; \bar{\alpha}]$ and $\bar{\alpha}(x)=x+1$. A contradiction once again follows from Propositions 7.1 and 2.2(c).

We turn now to a consideration of those automorphisms of $\mathbb{C}[x, y]$ which are not triangular. Again following [43], an element $\alpha$ of $A$ is called square if it is not conjugate to a triangular automorphism, and, following [11], $\alpha$ is a generalised Hénon automorphism if

$$
\alpha(x)=y ; \quad \alpha(y)=\lambda x+\beta(y)
$$

for some $\lambda \in \mathbb{C} \backslash\{0\}$ and $\beta(y) \in \mathbb{C}[y]$, with $\beta(y)$ having degree at least two. ${ }^{1}$ By [11, Theorem 2.6], every square element of $A$ is conjugate to a product of generalised Hénon automorphisms. $^{2}$

Let us examine first the prime spectrum of $S_{\mathbb{C}, 2, \alpha}$ when $\alpha$ is square. We follow initially in the proof of the following lemma the argument of [25, proof of Proposition 7.8, first paragraph].

Lemma 7.3 Let $\alpha$ be a square automorphism of $\mathbb{C}[x, y]$. Then $\operatorname{Spec}\left(S_{\mathbb{C}, 2, \alpha}\right)$ is the disjoint union of $\mathcal{V}(\theta):=\left\{P \in \operatorname{Spec}\left(S_{\mathbb{C}, 2, \alpha}\right): \theta \in P\right\}$, and $\mathcal{C}(\theta):=\operatorname{Spec}\left(S_{\mathbb{C}, 2, \alpha}\right) \backslash \mathcal{V}(\theta)$, and these sets have the following descriptions:

(a) $\mathcal{V}(\theta)$ is homeomorphic to $\operatorname{Spec}(\mathbb{C}[x, y])$.

(b) $\mathcal{C}(\theta)$ is homeomorphic to $\operatorname{Spec}\left(T_{\mathbb{C}, 2, \alpha}\right)$, where $T_{\mathbb{C}, 2, \alpha}:=\mathbb{C}[x, y]\left[\theta^{ \pm 1} ; \alpha\right]$.

(c) $\operatorname{Spec}\left(T_{\mathbb{C}, 2, \alpha}\right)$ is partitioned into the following three disjoint subsets.

(i) An uncountable set of co-Artinian maximal ideals $\left\{M_{j, \lambda}: j \in \mathbb{N}, \lambda \in \mathbb{C}\right\}$, all of height 2;

\footnotetext{
${ }^{1}$ This degree condition is mistakenly omitted from the definition in [25, page 368], although it is present in [11, Definition 2.5]. The condition omitted by [25] is definitely needed for the validity of some of the results in [11].

2 [11, Theorem 2.6] is slightly mis-stated, since the cyclically reduced automorphisms which it treats include all the square automorphisms, but also the affine automorphisms which are not also elementary, such as, for example the map $\tau: x \mapsto y, y \mapsto x$. See the definitions on pages 68-69 of [11]. In fact, [11, Theorem 2.6] is valid, in the terminology of that paper, for the elements of $A$ having degree at least 2.
} 
(ii) A countably infinite set of height one mutually comaximal prime ideals $\left\{P_{j}: j \in \mathbb{N}\right\}$, with

$$
\bigcap_{j \in \mathbb{N}} P_{j}=(0)
$$

(iii) (0).

(d) For each $j \in \mathbb{N}$, there exists a positive integer $n_{j}$ and a finite $\langle\alpha\rangle$-orbit $\left\{Q_{j, 0}, Q_{j, 1}=\right.$ $\left.\alpha\left(Q_{j, 0}\right), \ldots, Q_{j, n_{j}-1}=\alpha^{n_{j}-1}\left(Q_{j, 0}\right)\right\}$ of maximal ideals of $\mathbb{C}[x, y]$, such that

$$
P_{j}=\left(\bigcap_{\ell=0}^{n_{j}-1} Q_{j, \ell}\right) T_{\mathbb{C}, 2, \alpha} .
$$

(e) For $j \in \mathbb{N}$, denote the prime ideal $P_{j} \cap S_{\mathbb{C}, 2, \alpha}$ of $S_{\mathbb{C}, 2, \alpha}$ by $P_{j}^{\prime}$. Thus $P_{j}^{\prime}=$ $\left(\bigcap_{\ell=0}^{n_{j}-1} Q_{j, \ell}\right) S_{\mathbb{C}, 2, \alpha}$. For each $j \in \mathbb{N}$, (writing $\theta$ also for the image of $\theta$ in $S_{\mathbb{C}, 2, \alpha} / P_{j}^{\prime}$ and in $T_{\mathbb{C}, 2, \alpha} / P_{j}$ ),

$$
\left(S_{\mathbb{C}, 2, \alpha} / P_{j}^{\prime}\right)\left[\theta^{-1}\right]=T_{\mathbb{C}, 2, \alpha} / P_{j} \cong M_{n_{j}}\left(\mathbb{C}\left[\theta^{ \pm n_{j}}\right]\right) .
$$

In particular, $T_{\mathbb{C}, 2, \alpha} / P_{j}$ is an Azumaya algebra over its centre $\mathbb{C}\left[\theta^{ \pm n_{j}}\right]$, so the maximal ideals of $T_{\mathbb{C}, 2, \alpha} / P_{j}$ are parametrised by $\mathbb{C}$, and are the ideals

$$
\left\{M_{j, \lambda} / P_{j}: \lambda \in \mathbb{C}\right\} .
$$

Hence, for all $j \in \mathbb{N}$ and $\lambda \in \mathbb{C}$,

$$
T_{\mathbb{C}, 2, \alpha} / M_{j, \lambda} \cong M_{n_{j}}(\mathbb{C}) .
$$

(f) The integers $n_{j}$, for $j \in \mathbb{N}$, are unbounded; more precisely, every sufficiently large prime number occurs amongst the $n_{j}$.

Proof The partition of $\operatorname{Spec}\left(S_{\mathbb{C}, 2, \alpha}\right)$ into $\mathcal{V}(\theta)$ and $\mathcal{C}(\theta)$ is clear, and (a) and (b) follow at once since $S_{\mathbb{C}, 2, \alpha} / \theta S_{\mathbb{C}, 2, \alpha} \cong R$ and $T_{\mathbb{C}, 2, \alpha}$ is the localization of $S_{\mathbb{C}, 2, \alpha}$ with respect to powers of $\theta$.

By [11, Theorem 3.1], $\mathbb{C}[x, y]$ has countably infinitely many maximal ideals with a finite $\langle\alpha\rangle$-orbit. Enumerating these orbits by the parameter $j \in \mathbb{N}$, and letting $n_{j}$ be the size of the $j$ th orbit, the integers $n_{j}$ satisfy (f), by [11, Corollary 8.6 and note added in proof, page 97]. Labelling the $j$ th finite $\langle\alpha\rangle$-orbit as in (d), and defining the corresponding induced ideal $P_{j}$ also as in (d), yields a countably infinite set of comaximal prime ideals of $T_{2, \alpha, \mathbb{C}}$ by Lemma 2.3(b), giving the subset (c)(ii) of $\operatorname{Spec}\left(T_{\mathbb{C}, 2, \alpha}\right)$, see also [45, Theorem III.31]. The subset (c)(iii) is clear.

Let $I$ be any other prime ideal of $T_{2, \alpha, \mathbb{C}}$, so that $I$ is non-zero and

$$
I \cap \mathbb{C}[x, y]=\left(I \cap S_{\mathbb{C}, 2, \alpha}\right) \cap \mathbb{C}[x, y]
$$

is an $\alpha$-prime ideal of $\mathbb{C}[x, y]$ by Lemma 2.3(c). If $I \cap \mathbb{C}[x, y]$ is co-Artinian, then, by construction, $I$ contains one of the primes $P_{j}$ constructed above. Otherwise,

$$
I \cap \mathbb{C}[x, y]=(0),
$$

since $\mathbb{C}[x, y]$ has no $\alpha$-stable proper principal ideal, by [28]; see [43, note added in proof, page 572]. Assume now that (15) holds. By [21, Theorem 4.3], as $\alpha$ is of infinite order the 
only prime of $S_{\mathbb{C}, 2, \alpha}$, not containing $\theta$, which lies over (0) in $\mathbb{C}[x, y]$ is (0). Thus (0) is the only prime ideal of $T_{\mathbb{C}, 2, \alpha}$ not containing one of the ideals $P_{j}$.

It remains to prove (e), in the course of which the maximal ideals of (c)(i) will be described. It follows easily from Lemma $2.3(\mathrm{~b})$ that $T_{\mathbb{C}, 2, \alpha} / P_{j} \cong \bar{R}\left[\theta^{ \pm 1} ; \alpha\right]$ where $\bar{R}$ is the direct sum of $n_{j}$ copies of $\mathbb{C}$ and $\alpha$ acts on $\bar{R}$ by $\alpha\left(e_{i}\right)=e_{(i+1) \bmod n_{j}}$, where $\left\{e_{i}: 1 \leq i \leq n_{j}\right\}$ is the set of primitive idempotents of $\bar{R}$. Set $n=n_{j}, f=\left(1-e_{1}\right) \theta, a=\theta^{-n+1}$ and $b=\theta^{-1}$. Then: $f^{n-1}=\left(1-e_{1}\right)\left(1-e_{2}\right) \ldots\left(1-e_{n-1}\right) \theta^{n-1}=e_{n} \theta^{n-1}, a f^{n-1}=\theta^{-n+1} e_{n} \theta^{n-1}=e_{1}$ and $f b=1-e_{1}$. Thus $a f^{n-1}+f b=1$ and $f^{n}=0$. Therefore, by [1, Theorem 1.3], $R\left[\theta^{ \pm 1} ; \alpha\right]=M_{n}(B)$ with $e_{11}=a f^{n-1}=e_{1}$. As $\theta^{n}$ is central and $e_{1} \theta^{k} e_{1}=0$, for $0<k<n$, we have $B=e_{11} R\left[\theta^{ \pm 1} ; \alpha\right] e_{11}=\left(e_{1} R\right)\left[\theta^{ \pm n}\right] \simeq \mathbb{C}\left[\theta^{ \pm n}\right]$ and (14) holds. Now using standard arguments it is easy to complete the proof.

We can now summarise our (at present incomplete) knowledge about the occurrence of property $(\diamond)$ for the algebras $S_{\mathbb{C}, 2, \alpha}$. Recall that, by definition, every element of $\operatorname{Aut}_{\mathbb{C}-\operatorname{alg}}(\mathbb{C}[x, y])$ is either triangular or square.

Theorem 7.4 Let $R=\mathbb{C}[x, y]$, let $\alpha \in A=\operatorname{Aut}_{\mathbb{C}-\text { alg }}(R)$, and let $S_{\mathbb{C}, 2, \alpha}=R[\theta ; \alpha]$.

(a) Suppose that $\alpha$ is triangular. Then $S_{\mathbb{C}, 2, \alpha}$ satisfies $(\diamond)$ if and only if $\alpha$ has finite order if and only if $\alpha$ is conjugate to an element of type $(i)$, with $\lambda, \mu$ roots of unity in $\mathbb{C}$.

(b) Suppose that $\alpha$ is square. Then $S_{\mathbb{C}, 2, \alpha}$ satisfies $(\diamond)$ if and only if $S_{\mathbb{C}, 2, \alpha}$ is not primitive.

Proof (a) This is part of Lemma 7.2.

(b) Suppose that $S_{\mathbb{C}, 2, \alpha}$ is primitive. Then $S_{\mathbb{C}, 2, \alpha}$ does not satisfy ( $\left.\diamond\right)$ by Theorem 5.4(b).

Suppose on the other hand that $S_{\mathbb{C}, 2, \alpha}$ is not primitive. Then, from the description of the prime spectrum of $S_{\mathbb{C}, 2, \alpha}$ in Lemma 7.3, it is clear, bearing in mind Kaplansky's theorem [4, Theorem I.13.3], that the only primitive ideals of $S_{\mathbb{C}, 2, \alpha}$ are the co-Artinian maximal ideals $M_{j, \lambda}$. Hence, every simple $S_{\mathbb{C}, 2, \alpha}$-module is finite dimensional over $\mathbb{C}$. Therefore, $S_{\mathbb{C}, 2, \alpha}$ satisfies $(\diamond)$ by Theorem 6.1 .

Of course, Theorem 7.4(b) begs the following obvious question. Keep the notation of Theorem 7.4, and suppose that $\alpha$ is a square automorphism. Is $S_{\mathbb{C}, 2, \alpha}$ primitive? The simplest square automorphism is perhaps the map $x \mapsto y, y \mapsto x+y^{2}$. We do not even know the answer in this case.

Using the Leroy-Matczuk criterion for primitivity, Theorem 4.2, coupled with Lemma 7.3 , it is not hard to reduce the above question to one about the geometry of the finite orbits of square automorphisms, a question which may be of independent interest. It seems more natural to frame it in terms of points in the plane $\mathbb{C}^{2}$, rather than in terms of maximal ideals of $R$ :

Proposition 7.5 Keep the notation of Theorem 7.4, with $\alpha$ square. Denote the countably infinitely many finite $\langle\alpha\rangle$-orbits of points in $\mathbb{C}^{2}$ by $\mathcal{P}_{j}$, for $j \in \mathbb{N}$. Then $S_{\mathbb{C}, 2, \alpha}$ is primitive if and only if a point $\left(a_{j}, b_{j}\right) \in \mathcal{P}_{j}$ can be chosen, for every $j \in \mathbb{N}$, such that $\left\{\left(a_{j}, b_{j}\right): j \in \mathbb{N}\right\}$ lies on a (not necessarily irreducible) affine curve in $\mathbb{C}^{2}$.

We leave the straightforward proof to the reader and refer to [25, Lemma 2.6(v)] for an equivalent description of $\alpha$-special rings. 


\subsection{Subalgebras of group algebras over absolute fields}

This family of examples shows that neither of the conditions (c) $S$ satisfies a polynomial identity nor $(d)|\alpha|<\infty$ is implied by conditions $(a)$ or $(b)$ of Theorem 6.1. Note that $(c)$ and $(d)$ are equivalent when $R$ is semiprime, by [9] or [35].

Let $A$ be a free abelian group of finite rank $t,(t>1)$. Write $A$ multiplicatively with free generating set $X_{1}, \ldots, X_{t}$. Let $M \in G L_{t}(\mathbb{Z})$, and define an automorphism $\alpha$ of $A$ using $M$; that is, for $\mathbf{X}^{\mathbf{n}}=X_{1}^{n_{1}} \ldots X_{t}^{n_{t}} \in A$, with $\mathbf{n}=\left(n_{1}, \ldots, n_{t}\right) \in \mathbb{Z}^{t}$,

$$
\alpha\left(\mathbf{X}^{\mathbf{n}}\right):=\mathbf{X}^{M \mathbf{n}},
$$

where $\mathbf{n}$ is written as a column vector on the right hand side.

Let $\left\langle\theta^{ \pm 1}\right\rangle$ be an infinite cyclic group, and form the semidirect product

$$
G:=A \rtimes_{\alpha}\left\langle\theta^{ \pm 1}\right\rangle,
$$

so that $G$ is a torsion-free polycyclic group of Hirsch length $t+1$. Now let $p$ be a prime integer, and let $k$ be a subfield of the algebraic closure of the field of $p$ elements. By a celebrated result of Roseblade, [38], every simple module for the group algebra $k G$ has finite dimension as a $k$-vector space. (Roseblade's result applies to all polycyclic-by-finite groups; the case where the group is nilpotent-by-finite was proved by Philip Hall in 1959 [18].)

Let $S$ be the subalgebra $k A[\theta ; \alpha]$ of $k G$. We claim that $S$ inherits from $k G$ the property that all its simple modules are finite dimensional. To see this, let $W$ be a simple $S$-module. Since the powers of $\theta$ form an Ore set in $S, W$ is either $\theta$-torsion or $\theta$-torsion free. In the first case, $W \theta=0$, so that $W$ is a simple module over $S / \theta S \cong k A$. Therefore $\operatorname{dim}_{k}(W)<\infty$ by Hilbert's Nullstellensatz. In the second case, $W \theta=W$, so $W$ carries a structure as $S\left\langle\theta^{-1}\right\rangle$-module, that is as $k G$-module. As such, it is necessarily simple, and hence has finite $k$-dimension by Roseblade's theorem [38].

Finally, choosing $M$ to be a matrix of infinite order, we obtain an algebra $S$ which is a skew polynomial $k$-algebra over a Laurent polynomial coefficient algebra, which has property $(\diamond)$ but for which $|\alpha|=\infty$.

\subsection{A subalgebra of a group algebra in characteristic 0}

The following example is considered in [25, 7.10-7.14].

Let $S=R[\theta ; \alpha]$, where $R=\mathbb{C}\left[x^{ \pm 1}, y^{ \pm 1}\right]$ and $\alpha \in \operatorname{Aut}_{\mathbb{C}-\text { alg }}(R)$ is defined by $\alpha(y)=x$ and $\alpha(x)=y x^{-1}$. Note that $|\alpha|=\infty$. In [25], Jordan studies both the algebras $S:=R[\theta ; \alpha]$ and $T:=R\left[\theta^{ \pm 1} ; \alpha\right]$. He proves in [25, Proposition 7.13] that $T$ is primitive.

In the same proposition it is claimed that $R$ is not $\alpha$-special, so that, by the Leroy-Matczuk theorem, Theorem 4.2, $S$ is therefore not primitive. As is shown in [25, Propositions 7.11, 7.12], every non-zero primitive ideal of $S$ is co-Artinian. Hence, if $S$ itself is not primitive, then every simple $S$-module is finite dimensional, and $S$ satisfies $(\diamond)$ by Theorem 6.1. However, as we observed in the first version of the current paper, there is a gap in the proof that $R$ is not $\alpha$-special in [25, Proposition 7.13]. We are indebted to Ken Goodearl and to the referee, who independently provided arguments to repair the gap in Jordan's work. We give Goodearl's argument in Theorem 7.6. It is shorter than the referee's proof, although requiring the application of a result from [3]. The referee's argument is sketched in Remark 7.8. We thank both Goodearl and the referee for their input, and for permission to include the arguments below. 
Theorem 7.6 (Goodearl [15]) Let $\alpha$ be the automorphism of the variety $\left(\mathbb{C}^{\times}\right)^{2}$ given by $\alpha(a, b)=(b, a b)$. There does not exist any curve $C \subseteq\left(\mathbb{C}^{\times}\right)^{2}$ which meets every finite $\alpha$-orbit.

Proof Assume there does exist a curve $C \subseteq\left(\mathbb{C}^{\times}\right)^{2}$ which meets every finite $\alpha$-orbit.

As Jordan showed in [25, Proposition 7.11(ii)], for each prime $p \equiv 1(\bmod 5)$, there exists a finite $\alpha$-orbit $\mathcal{O}_{p}$ consisting of points $\left(\omega_{p}^{k^{m}}, \omega_{p}^{k^{m+1}}\right)$ for $m \in \mathbb{N}$, where $k$ is an integer satisfying $k^{2} \equiv k+1(\bmod \mathrm{p})$ and $\omega_{p}$ is a primitive $p$-th root of unity in $\mathbb{C}$. In particular, both coordinates of every point in $\mathcal{O}_{p}$ are primitive $p$-th roots of unity. By Dirichlet's Theorem, there are infinitely many primes $p \equiv 1(\bmod 5)$, so $\bigcup_{p \equiv 1(\bmod 5)} C \cap \mathcal{O}_{p}$ is infinite. Hence, there is at least one irreducible component $C_{i}$ of $C$ such that $\bigcup_{p \equiv 1(\bmod 5)} C_{i} \cap \mathcal{O}_{p}$ is infinite.

For any prime $p \equiv 1(\bmod 5)$, the orbit $\mathcal{O}_{p}$ is contained in the finite subgroup $H_{p}:=$ $\left\{\left(\omega_{p}^{i}, w_{p}^{j}\right) \mid i, j \in \mathbb{Z}\right\}$ of $\left(\mathbb{C}^{\times}\right)^{2}$. Consequently, there are infinitely many primes $p \equiv 1(\bmod 5)$ such that $C_{i} \cap\left(H_{p} \backslash\{(1,1)\}\right)$ is nonempty. Thus, the intersection of $C_{i}$ with the union of the finite subgroups of $\left(\mathbb{C}^{\times}\right)^{2}$ is infinite. By [3, Corollary 1], $C_{i}$ is contained in a proper algebraic subgroup $\Gamma$ of $\left(\mathbb{C}^{\times}\right)^{2}$.

Identify $\mathbb{C}\left[x^{ \pm 1}, y^{ \pm 1}\right]$ with $\mathcal{O}\left(\left(\mathbb{C}^{\times}\right)^{2}\right)$. Since $\Gamma$ is a proper closed subgroup of $\left(\mathbb{C}^{\times}\right)^{2}$, there exist integers $s$ and $t$, not both 0 , such that $\Gamma \subseteq V\left(1-x^{s} y^{t}\right)$. Hence, there are infinitely many primes $p \equiv 1(\bmod 5)$ such that $V\left(1-x^{s} y^{t}\right) \cap \mathcal{O}_{p}$ is nonempty.

Observe that $s^{2}+s t-t^{2} \neq 0$, and choose a prime $p \equiv 1(\bmod 5)$ not dividing $s^{2}+s t-t^{2}$, such that $V\left(1-x^{s} y^{t}\right) \cap \mathcal{O}_{p}$ is nonempty. Then $V\left(1-x^{s} y^{t}\right)$ contains a point $\left(\omega_{p}^{k^{m}}, \omega_{p}^{k^{m+1}}\right)$ with $m \in \mathbb{N}$ and $k \in \mathbb{Z}$ such that $k^{2} \equiv k+1(\bmod \mathrm{p})$. Now

$$
0=\left(1-x^{s} y^{t}\right)\left(\omega_{p}^{k^{m}}, \omega_{p}^{k^{m+1}}\right)=1-\omega_{p}^{(s+t k) k^{m}},
$$

whence $s+t k \equiv 0(\bmod \mathrm{p})$. Since $k^{2} \equiv k+1(\bmod \mathrm{p})$, it follows that $s^{2}+s t-t^{2} \equiv 0(\bmod \mathrm{p})$, contradicting our choice of $p$.

As claimed in the proof of [25, Proposition 7.13] (or as in Proposition 7.5), the non- $\alpha$ speciality in this example can be stated in geometric terms as in Theorem 7.6. Taking into account the previous discussion it follows that:

Corollary 7.7 Let $R=\mathbb{C}\left[x^{ \pm 1}, y^{ \pm 1}\right]$ and $\alpha \in \operatorname{Aut}_{\mathbb{C}-\text { alg }}(R)$ defined by $\alpha(y)=x$ and $\alpha(x)=$ $y x^{-1}$ and $S=R[\theta ; \alpha]$. Then $S$ is not primitive. Moreover, every simple $S$-module is finite dimensional, whereas $|\alpha|=\infty$. In particular, $S$ satisfies $(\diamond)$, but does not satisfy a polynomial identity.

Remark 7.8 Here is the promised sketch of the referee's patch for [25, Proposition 7.13]. With the help of the Nullstellensatz one can reduce to the case when the coefficients of the $\alpha$-special element $f$ belong to a finite Galois extension of $\mathbb{Q}$, then, replacing $f$ by $\prod_{\sigma \in G} f^{\sigma}$ where $G$ is the Galois group of the field extension, we may assume that $f$ has coefficients in $\mathbb{Q}$. Think of $\alpha$ as acting by the matrix $M=\left[\begin{array}{cc}-1 & 1 \\ 1 & 0\end{array}\right]$. By Dirichlet's Theorem and quadratic reciprocity there are infinitely many primes $p$ such that the polynomial $\operatorname{det}\left(M-\lambda I_{3}\right)$ splits $\bmod p$. For such a prime $p$ we can choose for $M$ an eigenvalue $c+p \mathbb{Z} \in \mathbb{Z} / p \mathbb{Z}$ and an eigenvector $\left(b_{1}+p \mathbb{Z}, b_{2}+p \mathbb{Z}\right)$ in $\mathbb{Z} / p \mathbb{Z} \times \mathbb{Z} / p \mathbb{Z}$. Now, for $\omega_{p}$ a primitive $p$-th root of unity, it follows that $\alpha^{-1}\left(\omega_{p}^{b_{1}}, \omega_{p}^{b_{2}}\right)=\left(\omega_{p}^{b_{1} c}, \omega_{p}^{b_{2} c}\right)$. Thus the $\alpha$-orbit of $\left(\omega_{p}^{b_{1}}, \omega_{p}^{b_{2}}\right)$ is contained in its Galois orbit. So $f(x, y)$ vanishes on these orbits. Therefore, for infinitely many primes $p$ there is at least one finite $\alpha$-orbit $\mathcal{O}_{p}$ of a point $\left(\omega_{p}^{e}, \omega_{p}^{g}\right)$ with $e, g$ not both zero $(\bmod p)$ 
such that $f(x, y)$ vanishes on every point of $\mathcal{O}_{p}$. Let $X$ be the Zariski closure of the union of those infinitely many $\mathcal{O}_{p}$ and $X_{1}, \ldots, X_{n}$ its one dimensional irreducible components. Since $\alpha$ permutes the $X_{i}$, for each $i$ there is an integer $m \geq 1$ such that each $X_{i}$ is stable under $\alpha^{m}$. Also as $X_{i}$ has a dense set of points that are periodic under $\alpha^{m}, \alpha^{m}$ has finite order on $X_{i}$. Let $N \geq 1$ be such that $\alpha^{N}$ is the identity on every $X_{i}$. Now $\alpha^{N}$ is the identity on each of the $\mathcal{O}_{p}$ for infinitely many $p$. Hence 1 is an eigenvalue of $M^{N}$, a contradiction.

\section{Questions}

For the convenience of the reader, we gather here a number of open questions arising from this work, some of them previously raised earlier in the text, some appearing here for the first time. As usual, $k$ is a field, and $S$ will denote the skew polynomial ring $R[\theta ; \alpha]$, where $R$ is commutative Noetherian and $\alpha$ is an automorphism. Further hypotheses will be added as needed.

First, a question which seeks to remove the gap between the necessary and sufficient conditions in Theorem $1.1(=5.4)$.

Question 8.1 Suppose that $S$ is primitive and satisfies $(\diamond)$. Must $R$ be a finite direct sum of fields?

We should note that the remaining case of Theorem 5.4 is the one where $\alpha$ is of infinite order, and $R$ is an $\alpha$-special Noetherian domain of Krull dimension 1 with countable spectrum. Moreover $R \mathcal{A}^{-1}$ is a field, where $\mathcal{A}$, as before, is the smallest $\alpha$-stable Ore set of $S$ containing an $\alpha$-special element. When $\mathcal{A}$ is finitely generated as a multiplicatively closed set (it is then possible to replace $\mathcal{A}$ by the set of powers of an $\alpha$-special element) $R$ is a $G$-domain (see [26, Theorem 19]). Thus, by [26, Theorem 156], $R$ is semilocal. The case when $R=K[[X]]$, the ring of formal power series and $\alpha$ is the automorphism given by $\alpha(X)=q X$, for $q \in K$ not a root of unity, is a key example to consider.

A second point where necessary and sufficient conditions fail to match is in the affine case, where the proof in one direction of Theorem $1.2(=6.1)$ needs the base field to be uncountable or of characteristic zero (cf. Proposition 5.3'). So we ask:

Question 8.2 Suppose that $R$ is an affine algebra over a countable field $k$ of nonzero characteristic and $S$ satisfies $(\diamond)$. Are all simple $S$-modules finite dimensional over $k$ ?

It is worth noting in connection with Question 8.2 that $(\diamond)$ is in general not well-behaved with regard to change of base field. Consider, for example, any polycyclic-by-finite group $G$ which is not abelian-by-finite; for instance, the Heisenberg group

$$
G=\langle x, y, z:[x, y]=z, z \text { central }\rangle .
$$

Choose a prime $p$, and let $k$ be the field of $p$ elements. Then $k G$ satisfies $(\diamond)$, as already noted in Sect. 1.3, thanks to Roseblade's theorems [38,39] (or by [18] if we take $G$ to be nilpotent as above). Now let $F$ be any field of characteristic $p$ which is not algebraic over $k$. Then $F G=F \otimes_{k} k G$ does not satisfy $(\diamond)$ by [33].

Haunting the results of Sect. 6 and the examples of Sect. 7 is the fundamental, if vague, question:

Question 8.3 Suppose that $R$ is $k$-affine and $\alpha \in \operatorname{Aut}_{k-a l g}(R)$. Are there "reasonable" necessary and sufficient hypotheses on $R$ and $\alpha$ which determine when all the simple $S$ modules are finite-dimensional? 
The examples stemming from group algebras considered in Sect. 7.3 show that the situation regarding Question 8.3 in positive characteristic is undoubtedly rather delicate. In characteristic 0 , Sect. 7.4 provides an example where $S$ is $k$-affine, has all its simple modules finite dimensional but does not satisfy a polynomial identity. Further such examples may be provided by the square automorphisms of $\mathbb{C}[x, y]$ studied in Sect. 7.2, where the question of primitivity remains open. We thus repeat the question asked after Theorem 7.4.

Question 8.4 Let $S=S_{\mathbb{C}, 2, \alpha}=\mathbb{C}[x, y][\theta ; \alpha]$, where $\alpha$ is a square automorphism. For example, $\alpha$ could be the map sending $x$ to $y$ and $y$ to $x+y^{2}$. Is $S$ primitive? Equivalently, does $S$ have an infinite dimensional simple module?

Acknowledgements Some of this work was done while the second author visited the University of Glasgow and the University of Warsaw, supported by Grant SFRH/BSAB/1286/2012. She would like to thank the two universities for their hospitality. The second author was also partially supported by CMUP (UID/MAT/00144/2013), which is funded by FCT (Portugal) with national (MEC) and European structural funds (FEDER), under the partnership agreement PT2020 and by the Warsaw Center of Mathematics and Computer Science. The first and third authors would like to thank the University of Porto and its staff for hospitality and good working conditions. The third author acknowledges the support of CMUP and of Polish National Center of Science Grant no. DEC-2011/03/B/ST1/04893. The first author was supported by Leverhulme Fellowship EM-2017-081/9. We are thankful to David Jordan, Christian Lomp, António Machiavelo and Carlos Rito for very helpful discussions regarding the behaviour of orbits in $\mathbb{C}^{2}$ under the action of automorphisms. We are particularly grateful to Jason Bell, Ken Goodearl and the referee for their careful reading, for many helpful comments, and for their proofs of Theorem 7.6, and for the remarks following Theorem 6.1 on the extension of that result to countable fields.

Open Access This article is distributed under the terms of the Creative Commons Attribution 4.0 International License (http://creativecommons.org/licenses/by/4.0/), which permits unrestricted use, distribution, and reproduction in any medium, provided you give appropriate credit to the original author(s) and the source, provide a link to the Creative Commons license, and indicate if changes were made.

\section{References}

1. Agnarsson, G., Amitsur, S.A., Robson, J.C.: Recognition of matrix rings. II. Isr. J. Math. 96, 1-13 (1996)

2. Bell, J.: Private communication

3. Bombieri, E., Masser, D., Zannier, U.: On unlikely intersections of compex varieties with tori. Acta Arith. 133, 309-323 (2008)

4. Brown, K.A., Goodearl, K.R.: Lectures on Algebraic Quantum Groups. Advanced Courses in Mathematics. CRM Barcelona, Birkhäuser Verlag, Basel (2002)

5. Brown, K.A., Warfield Jr., R.B.: The influence of ideal structure on representation theory. J. Algebra 116(2), 294-315 (1988)

6. Carvalho, P.A.A.B., Lomp, C., Pusat-Yilmaz, D.: Injective modules over down-up algebras. Glasg. Math. J. 52(A), 53-59 (2010)

7. Carvalho, P.A.A.B., Hatipoğlu, C., Lomp, C.: Injective hulls of simple modules over differential operator rings. Commun. Algebra 43(10), 4221-4230 (2015)

8. Carvalho, P.A.A.B., Musson, I.M.: Monolithic modules over Noetherian rings. Glasg. Math. J. 53(3), 683-692 (2011)

9. Damiano, R.F., Shapiro, J.: Twisted polynomial rings satisfying a polynomial identity. J. Algebra 92(1), 116-127 (1985)

10. Eisenbud, D.: Commutative Algebra with a View Toward Algebraic Geometry, Graduate Texts in Mathematics 150. Springer, Berlin (2004)

11. Friedland, S., Milnor, J.: Dynamical properties of plane polynomial automorphisms. Ergodic Theory Dyn. Syst. 9, 67-99 (1989)

12. Formanek, E., Jategaonkar, A.V.: Subrings of Noetherian rings. Proc. Am. Math. Soc. 46(2), 181-186 (1974)

13. Gilmer, R.: Multiplicative Ideal Theory, Queen's Papers in Pure and Applied Mathematics, vol. 90 (1992)

14. Goodearl, K.R.: Classical localizability in solvable enveloping algebras and Poincaré-Birkoff-Witt extensions. J. Algebra 132, 324-377 (1990) 
15. Goodearl, K. R.: Private communication

16. Goodearl, K.R., Warfield Jr., R.B.: An Introduction to Noncommutative Noetherian Rings, LMS Student Texts 61. Cambridge University Press, Cambridge (2004)

17. Hatipoğlu, C., Lomp, C.: Injective hulls of simple modules over finite dimensional nilpotent complex Lie superalgebras. J. Algebra 361, 79-91 (2012)

18. Hall, P.: On the finiteness of certain soluble groups. Proc. Lond. Math. Soc. (3) 9, 595-622 (1959)

19. Hirano, Y.: On injective hulls of simple modules. J. Algebra 225(1), 299-308 (2000)

20. Hrushowski, E.: The Elementary Theory of the Frobenius Automorphism. http://www.ma.huji.ac.il/ rehud/FROB.pdf

21. Irving, R.S.: Prime ideals of ore extensions over commutative rings. J. Algebra 56, 315-342 (1979)

22. Jategaonkar, A.V.: Jacobson's conjecture and modules over fully bounded Noetherian rings. J. Algebra 30, 103-121 (1974)

23. Jategaonkar, A.V.: Integral group rings of polycyclic-by-finite groups. J. Pure Appl. Algebra 4, 337-343 (1974)

24. Jategaonkar, A.V.: Localization in Noetherian rings. London Math. Soc. Lecture Note Series, vol. 98. Cambridge University Press, Cambridge (1986)

25. Jordan, D.A.: Primitivity in skew Laurent polynomial rings and related rings. Math. Z. 213(3), 353-371 (1993)

26. Kaplansky, I.: Commutative Rings, Revised edition. The University of Chicago Press Ltd., Chicago (1974)

27. Karamzadeh, O.A.S., Moslemi, B.: On G-type domains. J. Algebra Appl. 11(1), 1250005 (2012)

28. Lane, D.R.: PhD thesis, University of London (1976)

29. Leroy, A., Matczuk, J.: Primitivity of skew polynomial and skew Laurent rings. Commun. Algebra 24(7), 2271-2284 (1996)

30. Matlis, E.: Injective modules over Noetherian rings. Pacif. J. Math. 8, 511-528 (1958)

31. McConnell, J.C., Robson, J.C.: Noncommutative Noetherian Rings. Graduate Studies in Mathematics, vol. 30. American Mathematical Society, Providence, RI (2001)

32. Musson, I.M.: Injective modules for group rings of polycyclic groups, II. Q. J. Math Oxford Ser. 2(31), 449-466 (1980)

33. Musson, I.M.: Some examples of modules over Noetherian rings. Glasg. Math. J. 23, 9-13 (1982)

34. Musson, I.M.: Finitely generated, non-Artinian monolithic modules. In: New trends in Noncommutative Algebra (Seatle, WA, 2010) Contemp. Math., Amer. Math. Soc., , vol. 562, pp. 211-220. Providence

35. Pascaud, J.L., Valette, J.: Polynomes tordue a identite polynomiale. Commun. Algebra 16, 2415-2425 (1988)

36. Poole, D.G.: Localization in ore extensions of commutative noetherian rings. J. Algebra 128, 434-445 (1990)

37. Tanase, R.: Complex Hénon maps and discrete groups. Adv. Math. 295, 53-89 (2016)

38. Roseblade, J.E.: Group rings of polycyclic groups. J. Pure Appl. Algebra 3, 307-328 (1973)

39. Roseblade, J.E.: Applications to the Artin-Rees lemma to group rings, Sympos. Math. vol. 17, pp. 471-478 (convegno sui Gruppi Infiniti, INDAM, Rome 1973. Academic Press, London (1976)

40. Sant'ana, A., Vinciguerra, R.: On Cyclic Essential Extensions of Simple Modules Over Differential Operator Rings. arXiv:1704.04970v1

41. Sharp, R.Y., Vámos, P.: Baire's category theorem and prime avoidance in complete local rings. Arch. Math. 44, 243-248 (1985)

42. Smith, L.: Polynomial Invariants of Finite Groups, Research Notes in Mathematics, 6. A K Peters, Wellesley (1995)

43. Smith, M.: Eigenvectors of automorphisms of polynomial rings in two variables. Houst. J. Math. 10, 559-573 (1984)

44. Stenström, B.: Rings of Quotients. Springer, Berlin (1975)

45. Zariski, O., Samuel, P.: Commutative Algebra. D. Van Nostrand Company Inc, London (1958) 